\title{
La relación entre el gradiente socioeconómico y el consumo de cigarrillos en España
}

\section{The relationship between the socio-economic gradient and cigarette consumption in Spain}

\author{
Juan Manuel Martín Álvarez*, Jorge Barrientos Marín**, José María Millán***. \\ * Universidad Internacional de La Rioja. \\ ** Universidad de Antioquia. \\ *** Universidad de Huelva.
}

\section{Resumen}

El objetivo del estudio fue analizar la relación entre las características socioeconómicas individuales y el consumo de cigarrillos en España. La muestra estaba formada por 19.931 individuos de 15 o más años de edad de la Encuesta Europea de Salud en España (EESE) de 2014. Variables: prevalencia y nivel de consumo. Se realizó análisis de regresión multivariante logística ordinal con las variables socioeconómicas clase social, nivel educativo, actividad principal, situación económica y sector de actividad (solo para población trabajadora). Otras variables de control incluidas fueron las características sociodemográficas y los hábitos de vida saludables (ejercicio físico, alimentación y consumo de alcohol). Los factores que se relacionan con mayor prevalencia en el consumo de cigarrillos son: inferior clase social, no tener estudios universitarios, ser desempleado, tener peor situación económica y trabajar en hostelería. Por su parte, las variables relacionadas con el nivel de consumo de la población fumadora son: inferior clase social, no tener estudios universitarios, y no ser estudiante ni trabajador indefinido. En cuanto a las variables de control, aquellos regresores asociados a mayor prevalencia y nivel de consumo son: sexo masculino, edad entre 36 y 65 años, ser divorciado, menor número de niños en el hogar y peores hábitos de vida.

Palabras clave: Tabaquismo; cigarrillos; gradiente socioeconómico; hábitos de vida saludables; salud; Encuesta Europea de Salud.

\section{Abstract}

The objective of the study was to analyze the relationship between individual socioeconomic characteristics and cigarette consumption in Spain. The sample consisted of 19,931 individuals aged 15 or older who completed the European Health Interview Survey for Spain (EHSS-2014). Variables: prevalence and intensity of cigarette consumption. Multivariate ordered logistic regression analysis was performed with the following socioeconomic variables: social classes, educational attainment, main activity, economic situation and, for the working population, the activity sector. Other control variables were sociodemographic variables and healthy lifestyle habits (physical exercise, diet and alcohol consumption). The factors that relate to greater prevalence are: lower social class, not having university studies, being unemployed, having worse economic situation and working in hospitality industry. On the other hand, the variables related to higher intensity of cigarette consumption of the smoking population are: lower social class, not having university studies, and being neither a student nor on a permanent contract. Regarding control variables, those regressors associated with a higher prevalence and intensity of cigarette consumption are: being male, being aged between 36 and 65, being divorced, having fewer children at home and having worse lifestyle habits.

Keywords: Smoking; cigarettes; socio-economic gradient; lifestyle habits; health; European Health Interview Survey.

Recibido: Octubre 2019; Aceptado: Noviembre 2020.

Enviar correspondencia a:

José María Millán. Departamento de Economía, Universidad de Huelva, Facultad de Ciencias Empresariales y Turismo, Campus de La Merced, Plaza de la Merced 11, 21002, Huelva, España. Teléf.: (+34) 959217886.

E-mail: jmillan@uhu.es 


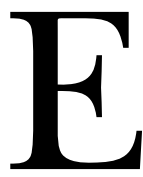

n España, según los datos de la última Encuesta Nacional de Salud de España 2017 (ENSE-2017), la prevalencia del consumo diario de tabaco en la población de 15 y más años es del 22,1\%, lo que indica que el hábito se ha reducido en más de 4 puntos porcentuales en la última década. Nótese en este sentido que la prevalencia de las olas previas en 2011-12 y 2006 fue de $23,9 \%$ y $26,4 \%$, respectivamente.

En cuanto al contexto internacional, la Figura 1 muestra la prevalencia de fumadores para todos los países de la UE-28 sobre la base de los datos de los dos últimos Eurobarómetros disponibles (números 429 y 458) sobre Actitudes de los europeos hacia el tabaco y los cigarrillos electrónicos para los periodos 2014 y 2017 (European Commission, 2014, 2017).

Como puede observarse, la prevalencia de fumadores en la UE-28 ha permanecido estable en torno al $26 \%$ para ambos periodos 2014 y 2017, si bien existen diferencias significativas entre países. También se observan disminuciones importantes en la prevalencia de fumadores en países como Bélgica (-6,2\%), Dinamarca (-4,4\%) o Suecia $(-4,3 \%)$, lo que coexiste con avances relevantes en esta prevalencia en países como Eslovaquia $(+5,6 \%)$, República Checa $(+4,5 \%)$ o Francia $(+4,1 \%)$.

En España, se observa una disminución en la prevalencia de fumadores en torno a 2 puntos porcentuales entre 2014 $(29,5 \%)$ y $2017(27,4 \%)$, si bien esta disminución viene precedida de un retroceso previo de 3,5 puntos entre 2012 $(33 \%)$ y $2014(29,5 \%)$, lo que ha hecho que España pase de ocupar el $4^{\circ}$ lugar en el ranking de la UE-27 en 2012 en cuanto a prevalencia en el consumo al $13^{\circ}$ puesto de la UE-28 en 2017 (European Commission, 2012, 2014, 2017).

Precisamente el alto coste sanitario y social del tabaquismo, unido al hecho de ser un factor de riesgo susceptible de prevención, ha convertido la reducción de la prevalencia de consumo del tabaco en uno de los objetivos prioritarios de las políticas de salud de cualquier país del entorno socioeconómico. En el caso de España, las campañas de información y sensibilización sobre los efectos del tabaco coexisten con: 1) la prohibición de fumar en lugares destinados al público, medios colectivos de transporte y lugares de trabajo; 2) la regulación en materia no solo de fabricación, presentación y venta de productos del tabaco sino de su propia publicidad y patrocinios; y 3) los fuertes impuestos a la elaboración y consumo de tabaco.

Estas intervenciones, unidas a la influencia de la crisis económica en su consumo (Martín-Álvarez, Golpe, Iglesias e Ingelmo, 2020), probablemente expliquen no solo la disminución de la prevalencia en el consumo de cigarrillos sino también los cambios en las conductas de consumo hacia otros productos del tabaco (en ocasiones más asequibles) como picadura de liar, puros, puritos o tabaco de pipa (López-Nicolás, Cobacho y Fernández, 2013). No es menos cierto, sin embargo, que estas intervenciones rara vez tienen en cuenta que el hábito tabáquico, a pesar de estar presente en todos los grupos sociales, no afecta por igual a toda la población (Almeida, Golpe, Iglesias y Martín-Álvarez, 2021).

Así, según el modelo de López, Collishaw y Piha (1994) de difusión de la epidemia en los países ricos, España se encuentra en la fase $I V$, caracterizada por una prevalencia en el consumo de tabaco con mayor nivel de concentración entre los grupos socioeconómicos más desfavorecidos. Esta influencia del gradiente socioeconómico en el tabaquismo se ha documentado suficientemente en la literatura académica internacional, mostrando una asociación entre consumo de tabaco y factores como desempleo, nivel educativo, tipo de ocupación y situación socioeconómica (Schaap, Van Agt y Kunst, 2008; White, Redner, Bunn y Higgins, 2016). En este sentido, la mayor prevalencia de hábitos de

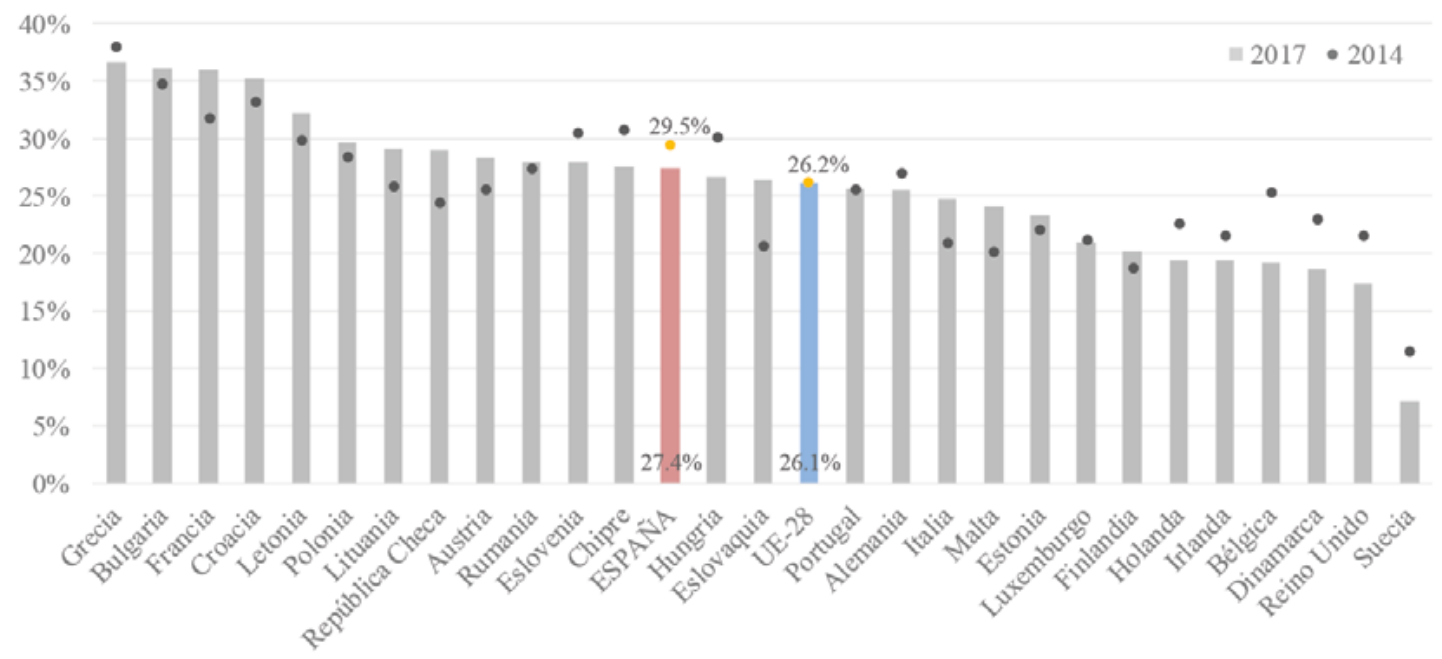

Figura 1. Prevalencia de fumadores (cigarrillos, puros, puritos o pipas) en la UE-28. Fuente. Eurobarómetros Especiales 429 (2014) y 458 (2017). Comisión Europea. 
vida poco saludables en aquellos grupos de menor nivel socioeconómico es uno de los mecanismos que vincula esta situación socioeconómica desfavorable con una peor salud (Macintyre, 1997). Más concretamente, el tabaquismo en aquellos grupos de menor nivel socioeconómico es la causa más importante de las diferencias socioeconómicas en la mortalidad (Stringhini et al., 2010).

Sin embargo, la generalización de los efectos de este gradiente hacia diferentes economías no es tan evidente y exige un respaldo basado en datos (Sarkar et al., 2017). Desafortunadamente, los trabajos que analizan estas asociaciones en clave nacional son prácticamente anecdóticos y, en ocasiones, sus resultados no están respaldados por modelos de regresión multivariantes sino tan solo por análisis descriptivos bivariantes (los trabajos de Agudo et al., 2004 y Pinilla y Abásolo, 2017 son notables excepciones).

Por tanto, corregir esta laguna de la literatura existente mediante la caracterización de la población de fumadores en España desde un punto de vista socioeconómico, e identificar así a los grupos de mayor riesgo de prevalencia y consumo, se convierte en objetivo prioritario desde la perspectiva de las autoridades sanitarias en aras de un diseño más efectivo de las políticas de control del tabaquismo, con objetivos más concretos, focalizados y más fácilmente evaluables.

Con ese objetivo, y utilizando los microdatos de la Encuesta Europea de Salud en España de 2014 (EESE-2014), este trabajo analiza la prevalencia en el consumo de cigarrillos y el nivel de consumo mediante técnicas de análisis bivariante y multivariante. En este sentido, el uso de la EESE-2014 permite generar información comparable a nivel europeo sobre la base de los datos más recientes disponibles. Por su parte, la elección del producto cigarrillos (que también incluye la picadura de liar) obedece a su incidencia todavía mayoritaria sobre el total del consumo, pese a los cambios detectados recientemente.

\section{Métodos}

\section{Instrumento del estudio}

Este trabajo utiliza los registros de participantes en la Encuesta Europea de Salud en España (EESE) 2014 (Ministerio de Sanidad, Servicios Sociales e Igualdad, 2015). La EESE, diseñada y coordinada por EUROSTAT, tiene como objetivo general proporcionar información sobre la salud de la población española de manera armonizada y comparable en el ámbito europeo con la finalidad de planificar y evaluar las actuaciones en materia sanitaria. Es una encuesta de diseño transversal, realizada con periodicidad quinquenal por el Instituto Nacional de Estadística (INE), en colaboración con el Ministerio de Sanidad, Servicios Sociales e Igualdad de España. El tipo de muestreo es trietápico estratificado, por secciones censales, viviendas familiares y personas. Los registros están disponibles para cualquier investigador en la web del INE en forma de fichero de microdatos anonimizados, y de acceso libre.

La EESE-2014 está estructurada en 2 cuestionarios: hogares y adultos. El cuestionario de hogares tan solo consta de un módulo sociodemográfico, mientras que el cuestionario de adultos consta de 4 módulos diferentes: 1) sociodemográfico; 2) estado de salud; 3) asistencia sanitaria; y 4) determinantes de la salud. Para el desarrollo de este trabajo se hizo uso de variables incluidas en ambos cuestionarios y en todos sus módulos.

El módulo de determinantes de la salud permitió disponer de información sobre el consumo de tabaco de los participantes (sección V), su alimentación (sección $\mathrm{U}$ ), su actividad física (sección T) y sus características físicas (sección S). En particular, este módulo permite la construcción de las siguientes variables utilizadas en el análisis: prevalencia en el consumo de cigarrillos, nivel de consumo de cigarrillos, tipo de alimentación, práctica de ejercicio físico en tiempo de ocio, consumo de alcohol e Índice de Masa Corporal -IMC-.

El módulo de asistencia sanitaria ofreció información sobre el seguro sanitario de los participantes (sección $\mathrm{O}$ ), que fue utilizada para generar la variable modalidad de seguro sanitario.

Por su parte, el módulo de estado de salud puso a disposición de este estudio la información sobre la salud mental de los participantes (sección G), lo que permitió generar la variable salud mental en los últimos 12 meses.

Finalmente, los módulos sociodemográficos, tanto en el cuestionario de hogares como de adultos, permitieron conocer, para cada participante, la composición de su hogar (sección A), sus características demográficas (sección E) y las características de su actividad económica (sección F). En concreto, de estos módulos surge la construcción de las variables sexo, edad, estado civil, número de niños en el hogar, clase social, nivel educativo, actividad principal y sector de actividad (solo para participantes con trabajo remunerado).

\section{Diseño y participantes}

El estudio ha sido de tipo observacional, epidemiológico y descriptivo. El objeto de estudio ha sido la totalidad de los registros de participantes de edad igual o superior a 15 años participantes en la EESE-2014, constituyendo un total de 22.842 registros. Se excluyen de la muestra final aquellos participantes que fuman de manera habitual productos distintos a los cigarrillos. No se excluye por tanto la picadura de liar; sí se excluyen los puros, tabaco de pipa y otros productos (apenas un 1\% del total de registros). Así mismo, se excluyen aquellos participantes que presentan valores no disponibles en variables relevantes para la 
realización de este análisis. La muestra final constituyó un total de 19.931 registros.

\section{Procedimiento}

De la información disponible en la EESE-2014 se seleccionaron como variables dependientes del estudio la prevalencia en el consumo de cigarrillos y el nivel de consumo. Para evaluar la prevalencia se consideraron 3 situaciones: 1) nunca fumador; 2) exfumador; y 3) fumador. Para evaluar el nivel de consumo se consideraron 4 niveles: 1) fumador ocasional; 2) fumador a diario de 10 o menos cigarrillos; 3) fumador a diario de entre 11 y 20 cigarrillos; y 4) fumador a diario de más de 20 cigarrillos.

Las variables independientes principales seleccionadas para el análisis fueron aquellas que guardan relación con el gradiente socioeconómico, esto es, clase social (basada en la ocupación del individuo o la persona de referencia), nivel educativo y variables económico-laborales como actividad principal, seguro de salud (únicamente sanidad pública excluyendo mutualidades del estado vs. seguro privado o mutualidades) y sector de actividad (CNAE-2009; solo para participantes con trabajo remunerado).

Las variables independientes de control fueron: 1) sociodemográficas-sexo, edad, estado civil y número de niños en el hogar-; 2) estado de salud-IMC y salud mental en los últimos 12 meses-; y 3) hábitos de vida saludable-práctica de ejercicio físico en tiempo de ocio, tipo de alimentación y consumo de alcohol-.

\section{Análisis estadístico}

El análisis estadístico de los datos se realizó con el programa Stata/MP-16 y consistió en un análisis descriptivo mediante el cálculo de recuentos (n) y proporciones $(\%)$ para las variables cualitativas y mediante el cálculo de media y desviación estándar para variables cuantitativas. También se realizó una comparación de proporciones de las variables categóricas mediante pruebas ji-cuadrado para tablas de contingencia. A fin de evaluar la prevalencia en el consumo de cigarrillos y el nivel de consumo, se realizaron 6 modelos de regresión multivariante logística ordinal, para los que se obtuvieron las razones de probabilidad $\mathrm{u}$ odds ratio (OR) con sus IC 95\%. La selección de variables independientes principales se llevó a cabo sobre la base del conocimiento previo de la relación existente entre el gradiente socioeconómico y el tabaquismo. La inclusión de variables independientes de control, para las que también existe una vinculación con el tabaquismo (tal y como se argumenta en la sección de «Discusión»), se fundamentó en un procedimiento de selección hacia delante (forward), sin que este proceso alterara de manera significativa los coeficientes asociados a las variables independientes principales. Todos los contrastes de hipótesis fueron bilaterales y la significación estadística se fijó en $\mathrm{p}<0,05$ ( 2 colas).

\section{Resultados}

\section{Análisis bivariante}

La Figura 2 muestra la información sobre la prevalencia en el consumo de cigarrillos y el nivel de consumo de los participantes en la muestra final.

La Tabla 1 muestra cómo varía este patrón de prevalencia y nivel de consumo para diferentes subgrupos poblacionales determinados en función de las variables independientes.

Los subgrupos más prevalentes dentro de la población de nunca fumadores ( $48 \%$ de la muestra final) son personas con educación básica (57\%), personas cuya actividad principal sea la de estudiante $(78 \%)$, labores del hogar $(66,3 \%)$, jubilado o prejubilado $(54,6 \%)$, trabajadores en el sector educativo $(52,6 \%)$, mujeres $(59,1 \%)$, personas con más de 65 años $(61,1 \%)$, y viudos $(70,5 \%)$.

En la población de exfumadores ( $28 \%$ de la muestra final) predominan personas de clase social I (31,3\%), incapacitados para trabajar $(37,4 \%)$, jubilados o prejubilados $(36,3 \%)$, empresarios o profesionales con asalariados $(32,5 \%)$, funcionarios $(32,4 \%)$, hombres $(36,6 \%)$, personas entre 51 y 65 años $(36,3 \%)$, y casados $(33,8 \%)$.

En cuanto a los fumadores ( $24 \%$ de la muestra final), los grupos más prevalentes son personas de clase social VI $(27,3 \%)$, personas con educación secundaria $(31,5 \%)$ y profesional $(31,1 \%)$, desempleados $(38,5 \%)$, trabajadores con contrato temporal $(33,7 \%)$, empresarios sin asalariados o trabajadores independientes $(32,9 \%)$, incapacitados para trabajar $(32,8 \%)$, trabajadores cuyos sectores de actividad sean, entre otros, hostelería $(38,2 \%)$, industria manufacturera $(35,6 \%)$ y construcción $(33,5 \%)$, hombres
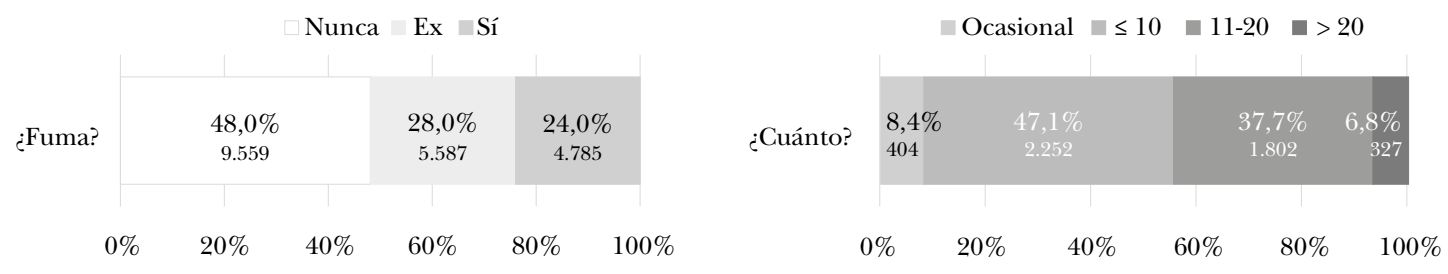

Figura 2. Prevalencia (panel izqdo.) y nivel de consumo (panel dcho.) de cigarrillos en España en 2014. Fuente. Elaboración propia con datos de la EESE-2014. 
Tabla 1. Prevalencia y nivel de consumo de cigarrillos en España en el año 2014 según diferentes características.

\begin{tabular}{|c|c|c|c|c|c|c|c|c|c|c|c|}
\hline & & \multirow{3}{*}{ n (\%) } & \multicolumn{3}{|c|}{ Prevalencia (\%) } & \multirow{3}{*}{ p } & \multicolumn{4}{|c|}{ Nivel de consumo (\%) } & \multirow{3}{*}{$\mathbf{p}$} \\
\hline & & & \multirow{2}{*}{ Nunca } & \multirow{2}{*}{ Ex } & \multirow{2}{*}{ Sí } & & \multirow{2}{*}{ Ocasional } & \multicolumn{3}{|c|}{ Fumador a diario } & \\
\hline & & & & & & & & $\leq 10$ & $11-20$ & $>20$ & \\
\hline & onsumo medio de cigarrillos $(1-80)^{a, b}$ & & & & & & & $\begin{array}{c}6,93 \\
(2,87) \\
\end{array}$ & $\begin{array}{c}17,5 \\
(3,04) \\
\end{array}$ & $\begin{array}{c}33,0 \\
(8,31) \\
\end{array}$ & \\
\hline \multicolumn{12}{|c|}{ Variables Independientes Principales -Gradiente Socioeconómico- } \\
\hline & lase social ${ }^{c}$ & & & & & $<0,001$ & & & & & $<0,001$ \\
\hline & Clase I (0-1) & $2.316(11,6 \%)$ & $47,6 \%$ & $31,3 \%$ & $21,1 \%$ & & $12,1 \%$ & $51,1 \%$ & $30,5 \%$ & $6,3 \%$ & \\
\hline & Clase II (0-1) & $1.173(8,6 \%)$ & $48,9 \%$ & $29,4 \%$ & $21,7 \%$ & & $9,2 \%$ & $51,7 \%$ & $33,7 \%$ & $5,4 \%$ & \\
\hline & Clase III (0-1) & $3.882(19,5 \%)$ & $47,6 \%$ & $28,7 \%$ & $23,7 \%$ & & $8,9 \%$ & $48,9 \%$ & $36,5 \%$ & $5,7 \%$ & \\
\hline & Clase IV (0-1) & $2.946(14,8 \%)$ & $47,0 \%$ & $29,9 \%$ & $23,1 \%$ & & $7,8 \%$ & $45,4 \%$ & $39,9 \%$ & $6,9 \%$ & \\
\hline & Clase V (0-1) & $6.387(32,0 \%)$ & $47,6 \%$ & $27,5 \%$ & $24,9 \%$ & & $7,1 \%$ & $46,9 \%$ & $38,8 \%$ & $7,2 \%$ & \\
\hline & ivel educativo & & & & & $<0,001$ & & & & & $<0,001$ \\
\hline & Educación básica (0-1) & $6.436(32,3 \%)$ & $57,0 \%$ & $27,6 \%$ & $15,4 \%$ & & $6,4 \%$ & $41,5 \%$ & $43,2 \%$ & $8,9 \%$ & \\
\hline & Educación secundaria (0-1) & $6.598(33,1 \%)$ & $41,0 \%$ & $27,5 \%$ & $31,5 \%$ & & $8,2 \%$ & $45,6 \%$ & $39,1 \%$ & $7,1 \%$ & \\
\hline & Educación profesional (0-1) & $2.934(14,7 \%)$ & $41,1 \%$ & $27,8 \%$ & $31,1 \%$ & & $8,7 \%$ & $49,2 \%$ & $36,7 \%$ & $5,4 \%$ & \\
\hline & Educación universitaria $(0-1)$ & $3.963(19.9 \%)$ & $49,9 \%$ & $29,8 \%$ & $20,3 \%$ & & $11,4 \%$ & $55,2 \%$ & $28,1 \%$ & $5,3 \%$ & \\
\hline \multicolumn{12}{|c|}{ Variables económico-laborales } \\
\hline & ctividad principal & & & & & $<0,001$ & & & & & $<0,001$ \\
\hline & Empresario o profesional con asalariados (0-1) & $532(2,7 \%)$ & $39,7 \%$ & $32,5 \%$ & $27,8 \%$ & & $12,2 \%$ & $35,8 \%$ & $42,6 \%$ & $9,4 \%$ & \\
\hline & $\begin{array}{l}\text { Empresario sin asalariados o } \\
\text { trabajador independiente }(0-1)\end{array}$ & $1.138(5,7 \%)$ & $37,7 \%$ & $29,4 \%$ & $32,9 \%$ & & $7,7 \%$ & $39,6 \%$ & $42,8 \%$ & $9,9 \%$ & \\
\hline & Funcionario (0-1) & $1.129(5,6 \%)$ & $46,0 \%$ & $32,4 \%$ & $21,6 \%$ & & $9,0 \%$ & $46,7 \%$ & $39,0 \%$ & $5,3 \%$ & \\
\hline & Trabajador indefinido (0-1) & $4.704(23,6 \%)$ & $41,0 \%$ & $28,4 \%$ & $30,6 \%$ & & $9,0 \%$ & $48,6 \%$ & $36,9 \%$ & $5,5 \%$ & \\
\hline & Trabajador con contrato temporal (0-1) & $1.313(6,6 \%)$ & $43,8 \%$ & $22,5 \%$ & $33,7 \%$ & & $9,5 \%$ & $47,2 \%$ & $37,4 \%$ & $5,9 \%$ & \\
\hline & Desempleado (0-1) & $2.717(13,6 \%)$ & $36,7 \%$ & $24,8 \%$ & $38,5 \%$ & & $8,4 \%$ & $46,0 \%$ & $37,8 \%$ & $7,8 \%$ & \\
\hline & Estudiante (0-1) & $1.077(5,4 \%)$ & $78,0 \%$ & $5,8 \%$ & $16,2 \%$ & & $17,2 \%$ & $69,5 \%$ & $12,1 \%$ & $1,2 \%$ & \\
\hline & Jubilado o prejubilado (0-1) & $5.115(25,7 \%)$ & $54,6 \%$ & $36,3 \%$ & $9,1 \%$ & & $3,9 \%$ & $47,0 \%$ & $41,4 \%$ & $7,7 \%$ & \\
\hline & Incapacitado para trabajar (0-1) & $369(1,8 \%)$ & $29,8 \%$ & $37,4 \%$ & $32,8 \%$ & & $7,4 \%$ & $39,7 \%$ & $40,5 \%$ & $12,4 \%$ & \\
\hline & Labores del hogar (0-1) & $1.604(8,0 \%)$ & $66,3 \%$ & $17,8 \%$ & $15,9 \%$ & & $6,3 \%$ & $51,4 \%$ & $37,6 \%$ & $4,7 \%$ & \\
\hline & Otra situación de inactividad (0-1) & $54(0,3 \%)$ & $37,0 \%$ & $31,5 \%$ & $31,5 \%$ & & $5,9 \%$ & $17,6 \%$ & $47,1 \%$ & $29,4 \%$ & \\
\hline & eguro de salud & & & & & $<0,001$ & & & & & 0,285 \\
\hline & $\begin{array}{l}\text { Únicamente sanidad pública } \\
\text {-no mutualidades del Estado- (0-1) }\end{array}$ & $15.850(79,5 \%)$ & $48,1 \%$ & $27,4 \%$ & $24,5 \%$ & & $8,1 \%$ & $46,9 \%$ & $38,0 \%$ & $7,0 \%$ & \\
\hline & $\begin{array}{l}\text { Mutualidades del Estado o } \\
\text { seguro privado }(0-1)\end{array}$ & $4.081(20,5 \%)$ & $47,5 \%$ & $30,5 \%$ & $22,0 \%$ & & $9,8 \%$ & $47,7 \%$ & $36,4 \%$ & $6,1 \%$ & \\
\hline \multicolumn{12}{|c|}{ Solo trabajadores } \\
\hline & tal & $8.995(45,1 \%)$ & $41,6 \%$ & $28,4 \%$ & $30,0 \%$ & & $8,9 \%$ & $46,2 \%$ & $38,4 \%$ & $6,5 \%$ & \\
\hline & ector de actividad (CNAE-2009) & & & & & $<0,001$ & & & & & $<0,001$ \\
\hline A & Agricultura, ganadería, silvicultura y pesca (0-1) & $415(4,6 \%)$ & $40,7 \%$ & $27,0 \%$ & $32,3 \%$ & & $10,4 \%$ & $33,6 \%$ & $47,8 \%$ & $8,2 \%$ & \\
\hline B & Industrias extractivas (0-1) & $28(0,3 \%)$ & $17,9 \%$ & $46,4 \%$ & $35,7 \%$ & & $20,0 \%$ & $20,0 \%$ & $60,0 \%$ & $0,0 \%$ & \\
\hline C & Industria manufacturera (0-1) & $1.155(12,8 \%)$ & $34,1 \%$ & $30,3 \%$ & $35,6 \%$ & & $9,0 \%$ & $43,1 \%$ & $41,3 \%$ & $6,6 \%$ & \\
\hline $\mathrm{D}$ & $\begin{array}{l}\text { Suministro de energía eléctrica, } \\
\text { gas, vapor y aire acondicionado (0-1) }\end{array}$ & $63(0,7 \%)$ & $39,7 \%$ & $33,3 \%$ & $27,0 \%$ & & $5,9 \%$ & $64,7 \%$ & $29,4 \%$ & $0,0 \%$ & \\
\hline E & $\begin{array}{l}\text { Suministro de agua, saneamiento, } \\
\text { residuos y descontaminación }(0-1)\end{array}$ & $74(0,8 \%)$ & $29,7 \%$ & $32,4 \%$ & $37,9 \%$ & & $10,7 \%$ & $46,4 \%$ & $32,2 \%$ & $10,7 \%$ & \\
\hline $\mathrm{F}$ & Construcción (0-1) & $493(5,5 \%)$ & $35,9 \%$ & $30,6 \%$ & $33,5 \%$ & & $4,3 \%$ & $40,6 \%$ & $41,2 \%$ & $13,9 \%$ & \\
\hline G & Comercio; reparación de vehículos (0-1) & $1.366(15,2 \%)$ & $41,4 \%$ & $26,2 \%$ & $32,4 \%$ & & $10,4 \%$ & $53,2 \%$ & $30,8 \%$ & $5,6 \%$ & \\
\hline $\mathrm{H}$ & Transporte y almacenamiento $(0-1)$ & $403(4,5 \%)$ & $35,7 \%$ & $31,3 \%$ & $33,0 \%$ & & $3,8 \%$ & $41,3 \%$ & $44,4 \%$ & $10,5 \%$ & \\
\hline 1 & Hostelería (0-1) & $647(7,2 \%)$ & $36,9 \%$ & $24,9 \%$ & $38,2 \%$ & & $8,1 \%$ & $39,7 \%$ & $44,5 \%$ & $7,7 \%$ & \\
\hline J & Información y comunicaciones (0-1) & $251(2,8 \%)$ & $48,6 \%$ & $22,3 \%$ & $29,1 \%$ & & $8,2 \%$ & $43,8 \%$ & $41,1 \%$ & $6,9 \%$ & \\
\hline
\end{tabular}




\begin{tabular}{|c|c|c|c|c|c|c|c|c|c|}
\hline M & $\begin{array}{l}\text { Actividades profesionales, } \\
\text { científicas y técnicas (0-1) }\end{array}$ & $435(4,8 \%)$ & $47,8 \%$ & $28,5 \%$ & $23,7 \%$ & $17,5 \%$ & $48,5 \%$ & $27,2 \%$ & $6,8 \%$ \\
\hline N & $\begin{array}{l}\text { Actividades administrativas } \\
\text { y servicios auxiliares }(0-1)\end{array}$ & $424(4,7 \%)$ & $39,4 \%$ & $27,6 \%$ & $33,0 \%$ & $7,1 \%$ & $53,6 \%$ & $35,7 \%$ & $3,6 \%$ \\
\hline 0 & $\begin{array}{l}\text { Admón. pública y defensa; } \\
\text { Seg. Social obligatoria }(0-1)\end{array}$ & $776(8,6 \%)$ & $42,4 \%$ & $32,2 \%$ & $25,4 \%$ & $9,6 \%$ & $42,7 \%$ & $41,6 \%$ & $6,1 \%$ \\
\hline$P$ & Educación (0-1) & $707(7,9 \%)$ & $52,6 \%$ & $26,9 \%$ & $20,5 \%$ & $5,5 \%$ & $55,2 \%$ & $34,5 \%$ & $4,8 \%$ \\
\hline Q & $\begin{array}{l}\text { Actividades sanitarias } \\
\text { y de servicios sociales (0-1) }\end{array}$ & $827(9,2 \%)$ & $43,3 \%$ & $30,7 \%$ & $26,0 \%$ & $7,9 \%$ & $48,4 \%$ & $39,5 \%$ & $4,2 \%$ \\
\hline $\mathrm{R}$ & $\begin{array}{l}\text { Actividades artísticas, recreativas } \\
\text { y de entretenimiento }(0-1)\end{array}$ & $160(1,8 \%)$ & $48,1 \%$ & $27,5 \%$ & $24,4 \%$ & $17,9 \%$ & $46,2 \%$ & $30,8 \%$ & $5,1 \%$ \\
\hline S & Otros servicios (0-1) & $193(2,1 \%)$ & $39,9 \%$ & $31,1 \%$ & $29,0 \%$ & $10,7 \%$ & $53,6 \%$ & $33,9 \%$ & $1,8 \%$ \\
\hline $\mathrm{T}$ & Actividades de los hogares (0-1) & $266(3,0 \%)$ & $54,5 \%$ & $20,7 \%$ & $24,8 \%$ & $10,6 \%$ & $51,5 \%$ & $34,9 \%$ & $3,0 \%$ \\
\hline U & $\begin{array}{l}\text { Actividades de organismos } \\
\text { extraterritoriales }(0-1)\end{array}$ & $5(0,1 \%)$ & $40,0 \%$ & $40,0 \%$ & $20,0 \%$ & $0,0 \%$ & $0,0 \%$ & $100,0 \%$ & $0,0 \%$ \\
\hline
\end{tabular}

Variables Independientes de Control

\begin{tabular}{|c|c|c|c|c|c|c|c|c|c|c|}
\hline \multicolumn{11}{|l|}{ Variables sociodemográficas } \\
\hline Sexo & & & & & $<0,001$ & & & & & $<0,001$ \\
\hline Mujer (0-1) & $9.399(47,2 \%)$ & $59,1 \%$ & $20,4 \%$ & $20,5 \%$ & & $8,8 \%$ & $53,2 \%$ & $34,0 \%$ & $4,0 \%$ & \\
\hline Hombre (0-1) & $10.532(52,8 \%)$ & $35,5 \%$ & $36,6 \%$ & $27,9 \%$ & & $8,2 \%$ & $42,0 \%$ & $40,6 \%$ & $9,2 \%$ & \\
\hline Edad (15-99) a & & $\begin{array}{c}52,0 \\
(20,3)\end{array}$ & $\begin{array}{c}55,3 \\
(15,6)\end{array}$ & $\begin{array}{c}44,9 \\
(13,4)\end{array}$ & & $\begin{array}{c}40,4 \\
(13,1)\end{array}$ & $\begin{array}{c}43,8 \\
(14,1)\end{array}$ & $\begin{array}{c}46,4 \\
(12,5)\end{array}$ & $\begin{array}{c}49,0 \\
(11,0)\end{array}$ & \\
\hline Edad & & & & & $<0,001$ & & & & & $<0,001$ \\
\hline $15-35$ años (0-1) & $4.006(20,1 \%)$ & $48,0 \%$ & $18,2 \%$ & $33,8 \%$ & & $12,1 \%$ & $53,5 \%$ & $31,9 \%$ & $2,5 \%$ & \\
\hline $36-50$ años $(0-1)$ & $6.204(31,1 \%)$ & $40,6 \%$ & $27,2 \%$ & $32,2 \%$ & & $7,9 \%$ & $45,1 \%$ & $40,2 \%$ & $6,9 \%$ & \\
\hline $51-65$ años (0-1) & $4.906(24,6 \%)$ & $38,0 \%$ & $36,3 \%$ & $25,7 \%$ & & $5,9 \%$ & $41,8 \%$ & $42,2 \%$ & $10,1 \%$ & \\
\hline Más de 65 años (0-1) & $4.815(24,2 \%)$ & $61,1 \%$ & $32,1 \%$ & $6,8 \%$ & & $4,5 \%$ & $50,3 \%$ & $37,9 \%$ & $7,3 \%$ & \\
\hline Estado civil & & & & & $<0,001$ & & & & & $<0,001$ \\
\hline Soltero $(0-1)$ & $5.209(26,1 \%)$ & $49,8 \%$ & $18,7 \%$ & $31,5 \%$ & & $9,9 \%$ & $50,5 \%$ & $34,1 \%$ & $5,6 \%$ & \\
\hline Casado (0-1) & $11.096(55,7 \%)$ & $44,4 \%$ & $33,8 \%$ & $21,8 \%$ & & $8,7 \%$ & $46,1 \%$ & $38,4 \%$ & $6,8 \%$ & \\
\hline Separado $(0-1)$ & $507(2,5 \%)$ & $36,1 \%$ & $28,4 \%$ & $35,5 \%$ & & $7,2 \%$ & $42,8 \%$ & $37,8 \%$ & $12,2 \%$ & \\
\hline Divorciado (0-1) & $937(4,7 \%)$ & $33,8 \%$ & $28,5 \%$ & $37,7 \%$ & & $4,0 \%$ & $39,4 \%$ & $47,0 \%$ & $9,6 \%$ & \\
\hline Viudo $(0-1)$ & $2.182(11,0 \%)$ & $70,5 \%$ & $20,4 \%$ & $9,1 \%$ & & $3,0 \%$ & $47,3 \%$ & $42,2 \%$ & $7,5 \%$ & \\
\hline Número de niños en el hogar $(0-6)^{a}$ & & $\begin{array}{c}0,39 \\
(0,74)\end{array}$ & $\begin{array}{c}0,39 \\
(0,74)\end{array}$ & $\begin{array}{c}0,45 \\
(0,75)\end{array}$ & & $\begin{array}{c}0,55 \\
(0,83)\end{array}$ & $\begin{array}{c}0,46 \\
(0,76)\end{array}$ & $\begin{array}{c}0,43 \\
(0,73)\end{array}$ & $\begin{array}{c}0,33 \\
(0,66)\end{array}$ & \\
\hline \multicolumn{11}{|l|}{ Hábitos de vida saludable } \\
\hline $\begin{array}{l}\text { Horas semanales de ejercicio físico } \\
\text { en tiempo de ocio }(0-50)\end{array}$ & & $\begin{array}{c}2,20 \\
(3,66)\end{array}$ & $\begin{array}{c}2,45 \\
(4,04)\end{array}$ & $\begin{array}{c}1,99 \\
(3,68)\end{array}$ & & $\begin{array}{c}2,72 \\
(4,35)\end{array}$ & $\begin{array}{c}2,27 \\
(3,87)\end{array}$ & $\begin{array}{c}1,67 \\
(3,36)\end{array}$ & $\begin{array}{c}0,93 \\
(2,53)\end{array}$ & \\
\hline Índice de alimentación saludable (-13 a 25) ${ }^{\mathrm{g}}$ & & $\begin{array}{c}11,2 \\
(5,05)\end{array}$ & $\begin{array}{c}11,4 \\
(4,92)\end{array}$ & $\begin{array}{c}8,87 \\
(5,76)\end{array}$ & & $\begin{array}{c}9,42 \\
(5,19)\end{array}$ & $\begin{array}{c}9,31 \\
(5,70)\end{array}$ & $\begin{array}{c}8,39 \\
(5,87)\end{array}$ & $\begin{array}{c}7,74 \\
(5,80)\end{array}$ & \\
\hline $\begin{array}{l}\text { Consumo medio diario de gramos } \\
\text { de alcohol puro }(0-185,71)^{\mathrm{h}}\end{array}$ & & $\begin{array}{c}3,03 \\
(7,14)\end{array}$ & $\begin{array}{c}6,95 \\
(10,8)\end{array}$ & $\begin{array}{c}7,42 \\
(12,5)\end{array}$ & & $\begin{array}{c}6,57 \\
(9,96)\end{array}$ & $\begin{array}{c}6,11 \\
(10,5)\end{array}$ & $\begin{array}{c}8,22 \\
(13,1)\end{array}$ & $\begin{array}{l}13,0 \\
(20,6)\end{array}$ & \\
\hline \multicolumn{11}{|l|}{ Estado de salud } \\
\hline Índice de Masa Corporal (1-4) a, e & & $\begin{array}{l}2,67 \\
(0,77)\end{array}$ & $\begin{array}{l}2,80 \\
(0,75)\end{array}$ & $\begin{array}{c}2,58 \\
(0,76)\end{array}$ & & $\begin{array}{c}2,55 \\
(0,76)\end{array}$ & $\begin{array}{c}2,50 \\
(0,72)\end{array}$ & $\begin{array}{c}2,62 \\
(0,76)\end{array}$ & $\begin{array}{c}2,90 \\
(0,83)\end{array}$ & \\
\hline Salud mental (últimos 12 meses) & & & & & $<0,001$ & & & & & $<0,001$ \\
\hline Enfermedad / problema de salud mental $(0-1)^{f}$ & $2.402(12,1 \%)$ & $50,4 \%$ & $24,0 \%$ & $25,6 \%$ & & $6,5 \%$ & $40,6 \%$ & $38,9 \%$ & $14,0 \%$ & \\
\hline Mentalmente sano (ref.) $(0-1)$ & $17.529(87,9 \%)$ & $47,6 \%$ & $28,6 \%$ & $23,8 \%$ & & $8,7 \%$ & $48,0 \%$ & $37,5 \%$ & $5,8 \%$ & \\
\hline
\end{tabular}

Nota. a) Variable cuantitativa. La información reportada es la media y la desviación estándar. b) Información solo disponible para fumadores a diario. c) Variable derivada basada en la ocupación de la persona de referencia: I, Directores/as y gerentes de establecimientos de 10 o más asalariados/as y profesionales tradicionalmente asociados/as a las licenciaturas universitarias; II, Directores/as y gerentes de establecimientos de menos de 10 asalariados/as y profesionales tradicionalmente asociados/as a diplomaturas universitarias y otros/as profesionales de apoyo técnico. Deportistas y artistas; III, Ocupaciones intermedias y trabajadores/as por cuenta propia; IV, Supervisores/as y trabajadores/as en ocupaciones técnicas cualificadas; V, Trabajadores/as cualificados/as del sector primario y otros/as trabajadores/as semi-cualificados/as; VI, Trabajadores/as no cualificados/as. d) Incluye trabajadores con contrato verbal o sin contrato, ayuda familiar, miembros de una cooperativa y otras situaciones. e) Esta variable, de tipo ordenada discreta, toma valores entre 1 y 4 y captura si la relación peso-altura del individuo, medida a través del valor de su IMC dentro de la escala propuesta por la International Obesity Task Force (IOFT), se clasifica como peso insuficiente (IMC $<18,5 ;$ valor de la variable IMC = 1), normopeso $(18,5 \leq I M C<25$; valor de la variable IMC $=2)$, sobrepeso $(25 \leq I M C<30$; valor de la variable IMC $=3)$ u obesidad (IMC $\geq 30$; valor de la variable IMC = 4). f) Depresión, ansiedad crónica u otros problemas mentales. g) Este índice se calcula sumando las frecuencias de consumo de fruta fresca (excluyendo zumos), zumo natural de frutas o verduras, verduras, ensaladas y hortalizas, legumbres y productos lácteos, y restando a su vez las frecuencias de consumo de dulces, refrescos con azúcar, comida rápida y aperitivos o comidas saladas de picar. La frecuencia de cada uno de estos grupos de alimentos se mide según la siguiente escala: $0=$ nunca, $1=$ menos de una vez a la semana, 2 = una o dos veces a la semana, 3 = tres veces a la semana, $4=$ de 4 a 6 veces a la semana, $5=$ una o más veces al día. h) La equivalencia en gramos de alcohol puro utilizada es: cerveza con alcohol, $10 \mathrm{~g}$ por unidad de bebida; vino o cava, $10 \mathrm{~g}$ por unidad de bebida; aperitivos con alcohol (vermut, fino, jerez), $20 \mathrm{~g}$ por unidad de bebida; licores, anís, pacharán, $20 \mathrm{~g}$ por unidad de bebida; whisky, coñac, combinados, $20 \mathrm{~g}$ por unidad de bebida; bebidas locales (sidra, carajillo...), $10 \mathrm{~g}$ por unidad de bebida; $\mathrm{p}$ = significación de la prueba ji-cuadrado. Fuente. elaboración propia con datos de la EESE-2014. 
(27,9\%), personas entre 15 y 35 años $(33,8 \%)$ y entre 36 y 50 años $(32,2 \%)$, divorciados $(37,7 \%)$, separados $(35,5 \%)$, y solteros $(33,8 \%)$.

Ya dentro de la población de fumadores, entre los fumadores ocasionales (8,4\% de la muestra final de fumadores) predominan personas de clase social I $(12,1 \%)$, personas con educación universitaria $(11,4 \%)$, estudiantes $(17,2 \%)$, empresarios o profesionales con asalariados (12,2\%), trabajadores cuyos sectores de actividad sean actividades artísticas, recreativas y de entretenimiento (17,9\%) y actividades profesionales, científicas y técnicas $(17,5 \%)$, y personas entre 15 y 35 años $(12,1 \%)$.

En cuanto a los fumadores a diario de más de 20 cigarrillos (6,8\% de la muestra final de fumadores), tienen mayor representación incapacitados para trabajar (12,4\%), empresarios sin asalariados o trabajadores independientes $(9,9 \%)$, empresarios o profesionales con asalariados $(9,4 \%)$, trabajadores cuyos sectores de actividad sean construcción $(13,9 \%)$ y transporte y almacenamiento $(10,5 \%)$, hombres $(9,2 \%)$, personas entre 51 y 65 años $(10,1 \%)$, separados $(12,2 \%)$, y divorciados $(9,6 \%)$.

En relación con las variables cuantitativas, los fumadores a diario de más de 20 cigarrillos (frente a los fumadores a diario de 10 o menos cigarrillos y fumadores ocasionales) presentan mayor IMC, mayor consumo diario de alcohol, menores horas semanales de ejercicio físico, menor índice de alimentación saludable e inferior número de niños en el hogar.

\section{Análisis multivariante}

Los resultados asociados al análisis multivariante de la prevalencia en el consumo de cigarrillos se presentan en los 4 modelos de regresión 1A, 1B, 2A y 2B de la Tabla 2. Para evaluar la prevalencia se consideraron 3 situaciones: 1) nunca fumador; 2) exfumador; y 3) fumador. La naturaleza ordenada del modelo utilizado genera 2 salidas en cada regresión: 1) situaciones 3-2 frente a 1 fumador y exfumador (frente a nunca fumador)-; y 2) situación 3 frente a 2-1 -fumador (frente a exfumador y nunca fumador)-. Precisamente la naturaleza ordenada del modelo hace que, en cada una de las 2 salidas en la regresión, la situación o situaciones alternativas (situación 1 o situaciones 2-1) sean, desde el punto de vista sanitario, más convenientes que las situaciones o situación principal (situaciones 3-2 o situación 3).

Los modelos 1A y 1B incluyen la totalidad de la muestra final (19.931) mientras que los modelos 2A y 2B tan solo incorporan a la población trabajadora (8.995). Los modelos tipo A capturan la información del gradiente socioeconómico a través de la clase social. Los modelos tipo B, por su parte, sustituyen la información compacta sobre el gradiente socioeconómico que captura la clase social por aquellas variables directamente relacionadas con este gradiente: nivel educativo y variables económico- laborales como actividad principal, seguro de salud -como "proxy" de la situación económica- y sector de actividad (solo modelo 2B).

En primer lugar se analizan los resultados asociados a la probabilidad de encontrar población fumadora y exfumadora (frente a nunca fumadora), que se muestran en el panel izquierdo de la Tabla 2. En relación con las variables asociadas al gradiente socioeconómico, el modelo 1A indica cómo esta probabilidad para la muestra total aumenta en las clases sociales IV $(\mathrm{OR}=1,13), \mathrm{V}(\mathrm{OR}=1,20)$ y VI $(\mathrm{OR}=1,19)$ frente a la clase social I. En el modelo $1 \mathrm{~B}$ esta probabilidad aumenta para la población con educación secundaria $(\mathrm{OR}=1,52)$ y profesional $(\mathrm{OR}=1,35)$ frente a la población con educación universitaria. En cuanto a la actividad principal (modelo 1B), y frente a la situación de empresario sin asalariados o trabajador independiente, esta probabilidad aumenta en incapacitados para trabajar $(\mathrm{OR}=1,49)$, desempleados $\quad(\mathrm{OR}=1,30)$, jubilados $\mathrm{o}$ prejubilados $(\mathrm{OR}=1,28)$ y disminuye para estudiantes $(\mathrm{OR}=0,24)$ y personas que realizan labores del hogar $(\mathrm{OR}=0,66)$. Por su parte, disponer únicamente de sanidad pública (modelo 1B) aumenta esta probabilidad $(\mathrm{OR}=1,10)$.

$\mathrm{Al}$ analizar esta probabilidad para la muestra de trabajadores, los modelos 2Ay 2B muestran, respectivamente, mayores efectos de las clases sociales y el nivel educativo que el observado para la muestra total en los modelos 1A y 1B. En relación con el sector de actividad, y frente a los trabajadores en hostelería, el modelo 2B muestra cómo esta probabilidad disminuye especialmente para trabajadores cuyos sectores sean actividades de los hogares $(\mathrm{OR}=0,52)$, actividades artísticas, recreativas y de entretenimiento $(\mathrm{OR}=0,59)$, información y comunicaciones $(\mathrm{OR}=0,61)$ y educación $(\mathrm{OR}=0,68)$.

En cuanto a las variables independientes de control, los modelos 1A y 1B muestran para la muestra total cómo esta probabilidad se reduce en mujeres, aumenta en personas de entre 36 y 65 años (frente a personas de entre 15 y 35 años), aumenta para separados y divorciados y disminuye para viudos (frente a solteros), aumenta para la población con peores hábitos (menor ejercicio físico, alimentación menos saludable, mayor consumo de alcohol), disminuye para personas con menor IMC, y aumenta para personas con alguna enfermedad o problema de salud mental. Los modelos 2A y 2B, por su parte, muestran resultados similares para la población trabajadora.

Los resultados relacionados con la probabilidad de encontrar población fumadora (frente a exfumadora y nunca fumadora) se muestran en el panel derecho de la Tabla 2. En relación con las variables asociadas al gradiente socioeconómico, el modelo 1A muestra cómo esta probabilidad para la muestra total aumenta significativamente en las clases sociales III $(\mathrm{OR}=1,16)$, IV $(\mathrm{OR}=1,23), \mathrm{V}(\mathrm{OR}=1,32)$ y VI $(\mathrm{OR}=1,47)$ frente a la clase social I. En el modelo $1 \mathrm{~B}$ esta probabilidad 


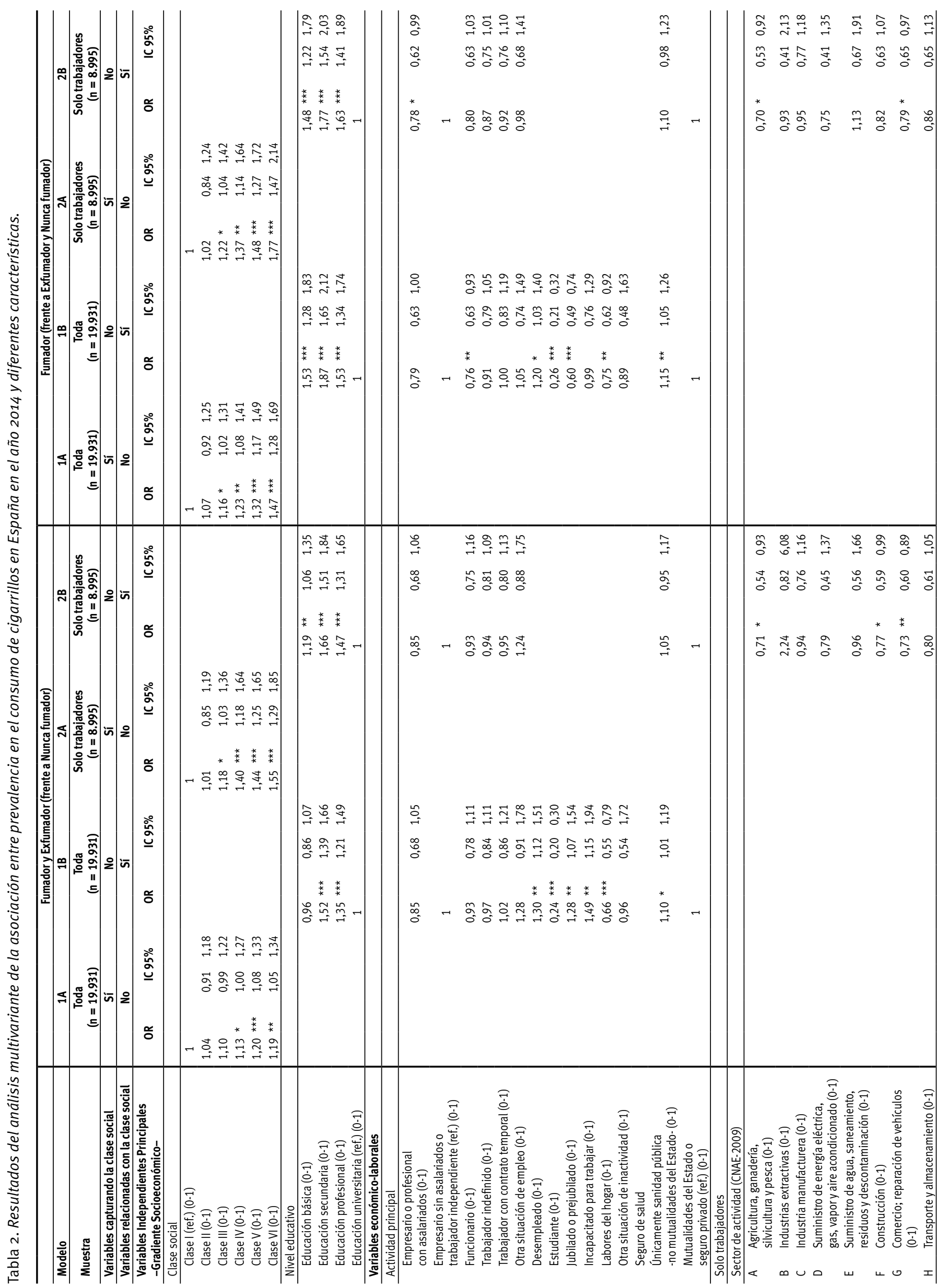




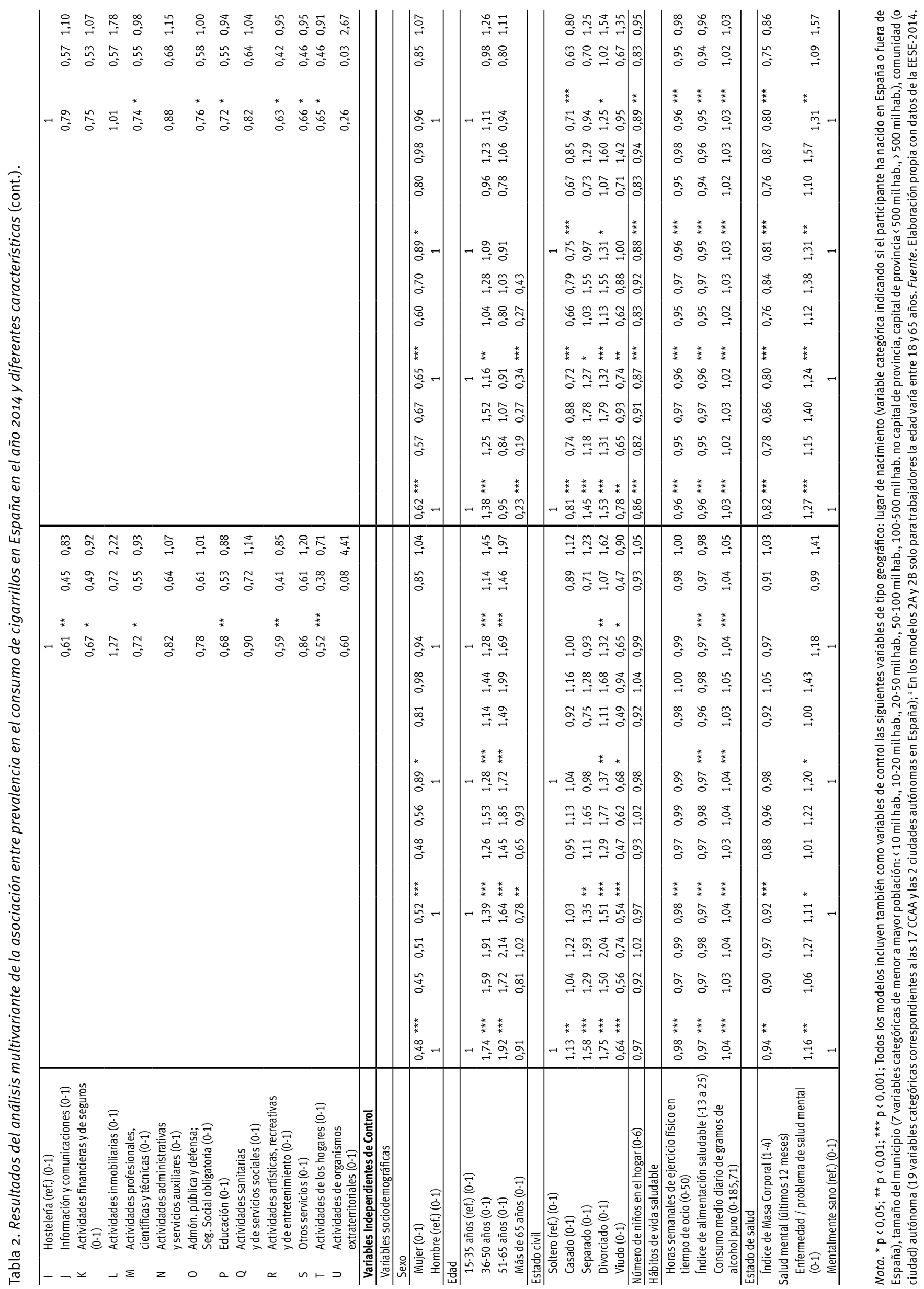


aumenta paralapoblación con educación básica $(\mathrm{OR}=1,53)$, secundaria $(\mathrm{OR}=1,87)$ y profesional $(\mathrm{OR}=1,53)$, frente a la población con educación universitaria. En cuanto a la actividad principal (modelo 1B), y frente a la situación de empresario sin asalariados o trabajador independiente, esta probabilidad aumenta para desempleados $(\mathrm{OR}=1,20)$ mientras que disminuye para estudiantes $(\mathrm{OR}=0,26)$, jubilados o prejubilados $(\mathrm{OR}=0,60)$, personas que realizan labores del hogar $(\mathrm{OR}=0,75)$ y funcionarios $(\mathrm{OR}=0,76)$. Por otro lado, disponer únicamente de sanidad pública (modelo 1B) aumenta esta probabilidad $(\mathrm{OR}=1,15)$.

$\mathrm{Al}$ analizar esta probabilidad para la muestra de trabajadores, los modelos 2A y 2B muestran, respectivamente, mayores efectos de las clases sociales y similares efectos del nivel educativo que el observado para la muestra total en los modelos 1A y 1B. En cuanto a la actividad principal (modelo 2B), y frente a la situación de empresario sin asalariados o trabajador independiente, esta probabilidad disminuye para empresarios o profesionales con asalariados $(\mathrm{OR}=0,78)$. En lo relativo al sector de actividad (modelo 2B), y frente a los trabajadores en hostelería, esta probabilidad disminuye significativamente para trabajadores en actividades artísticas, recreativas y de entretenimiento $(\mathrm{OR}=0,63)$, actividades de los hogares $(\mathrm{OR}=0,65)$, otros servicios $(\mathrm{OR}=0,66)$, agricultura, ganadería, silvicultura y pesca $(\mathrm{OR}=0,70)$, educación $(\mathrm{OR}=0,72), \quad \mathrm{y}$ actividades profesionales, científicas y técnicas $(\mathrm{OR}=0,74)$.

En lo relativo a las variables independientes de control, los modelos 1A y 1B muestran para la muestra total cómo esta probabilidad se reduce en mujeres, aumenta en personas de entre 36 y 50 años y disminuye en personas de más de 65 años (frente a personas de entre 15 y 35 años), aumenta para divorciados y separados, y disminuye para casados y viudos (frente a solteros), aumenta para la población con hábitos menos saludables, disminuye para personas con menor IMC, y aumenta para personas con alguna enfermedad o problema de salud mental. Los modelos 2A y 2B, por su parte, muestran resultados similares para la población trabajadora.

Los resultados asociados al análisis multivariante del nivel de consumo de cigarrillos de la muestra total de fumadores se presentan en los modelos 3A y 3B de la Tabla 3 (4.785 registros). Para evaluar el nivel de consumo, se consideraron 4 niveles: 1) fumador ocasional; 2) fumador a diario de 10 o menos cigarrillos; 3) fumador a diario de entre 11 y 20 cigarrillos; y 4) fumador a diario de más de 20 cigarrillos. La naturaleza ordenada del modelo utilizado genera 3 salidas en cada regresión: 1) niveles 4-3-2 vs. 1 -fumador a diario (vs. fumador ocasional); 2) niveles 4-3 vs. 2-1 -fumador a diario de más de 10 cigarrillos (vs. fumadores a diario de 10 o menos cigarrillos y fumadores ocasionales)-; y 3) nivel 4 vs. 3-2-1 -fumador a diario de más de 20 cigarrillos (vs. fumadores a diario de 20 o menos cigarrillos y fumadores ocasionales)-. También en este caso la naturaleza ordenada del modelo hace que, en cada una de las 3 salidas en la regresión, el nivel o los niveles alternativos (nivel 1, niveles 2-1 o niveles 3-2-1) sean más convenientes que los niveles o el nivel principal (niveles 4-3-2, niveles 4-3 o nivel 4) desde el punto de vista sanitario.

De manera análoga a la Tabla 2, el modelo 3A captura la información del gradiente socioeconómico a través de la clase social mientras que el 3B incluye aquellas variables directamente relacionadas con este gradiente: nivel educativo y variables económico-laborales como actividad principal y seguro de salud.

En primer lugar se analizan los resultados relacionados con la probabilidad de encontrar población fumadora a diario (frente a fumadora ocasional), que se presentan en el panel izquierdo de la Tabla 3. En lo relativo a las variables asociadas al gradiente socioeconómico, el modelo 3A indica cómo esta probabilidad aumenta para las clases sociales III $(\mathrm{OR}=1,48), \mathrm{IV}(\mathrm{OR}=1,64), \mathrm{V}(\mathrm{OR}=1,99)$ y $\mathrm{VI}(\mathrm{OR}=1,68)$ frente a la clase social I. En el modelo 3B, por su parte, se observa cómo, frente a la población con educación universitaria, esta probabilidad aumenta para la población con educación básica $(\mathrm{OR}=1,49)$ y secundaria $(\mathrm{OR}=1,48)$. En cuanto a la actividad principal, y frente a la situación de empresario sin asalariados o trabajador independiente, se observa cómo esta probabilidad disminuye para aquellas personas cuya actividad principal es la de estudiante $(\mathrm{OR}=0,51)$.

Con respecto a las variables independientes de control, los modelos 3A y 3B muestran cómo esta probabilidad aumenta en personas de entre 36 y 65 años (frente a personas de entre 15 y 35 años) y en divorciados (frente a solteros), así como para la población que menos ejercicio físico realiza y menos saludable es su alimentación.

A continuación se analizan los resultados relacionados con la probabilidad de encontrar población fumadora a diario de más de 10 cigarrillos (frente a fumadora a diario de 10 o menos cigarrillos y fumadores ocasionales), que se presentan en el panel central de la Tabla 3. Con respecto a las variables asociadas al gradiente socioeconómico, el modelo 3A indica cómo esta probabilidad aumenta significativamente en las clases sociales IV $(\mathrm{OR}=1,36), \mathrm{V}(\mathrm{OR}=1,34)$ y VI $(\mathrm{OR}=1,50)$ frente a la clase social I. En el modelo 3B, frente a la población con educación universitaria, esta probabilidad aumenta para la población con educación básica $(\mathrm{OR}=1,84)$, secundaria $(\mathrm{OR}=1,66)$ y profesional $(\mathrm{OR}=1,55)$. En cuanto a la actividad principal (modelo 3B), y frente a la situación de empresario sin asalariados o trabajador independiente, esta probabilidad disminuye para estudiantes $(\mathrm{OR}=0,20)$, trabajadores indefinidos $(\mathrm{OR}=0,72)$ y desempleados $(\mathrm{OR}=0,73)$.

En relación con las variables independientes de control, los modelos 3A y $3 \mathrm{~B}$ muestran cómo esta probabilidad se reduce en mujeres y en hogares con mayor número 
La relación entre el gradiente socioeconómico y el consumo de cigarrillos en España

Tabla 3. Resultados del análisis multivariante de la asociación entre nivel de consumo de cigarrillos en España en el año 2014 y diferentes características.

\begin{tabular}{|c|c|c|c|c|c|c|c|c|c|c|c|c|c|}
\hline \multirow[b]{2}{*}{ Modelo } & \multicolumn{4}{|c|}{ Fumador a diario (frente a Fumador ocasional) } & \multicolumn{5}{|c|}{$\begin{array}{l}\text { Fumador a diario }>10 \text { cigarrillos (frente a } \\
\text { Fumador a diario } \leq 10 \text { y Fumador ocasional) }\end{array}$} & \multicolumn{4}{|c|}{$\begin{array}{l}\text { Fumador a diario }>20 \text { cigarrillos (frente a } \\
\text { Fumador a diario } \leq 20 \text { y Fumador ocasional) }\end{array}$} \\
\hline & \multicolumn{2}{|c|}{$3 A$} & \multicolumn{2}{|c|}{ 3B } & \multicolumn{2}{|c|}{$3 A$} & \multicolumn{3}{|c|}{ 3B } & \multicolumn{2}{|c|}{$3 A$} & \multicolumn{2}{|r|}{ 3B } \\
\hline Muestra & \multicolumn{2}{|c|}{$\begin{array}{l}\text { Solo fumadores } \\
(n=4.785)\end{array}$} & \multicolumn{2}{|c|}{$\begin{array}{l}\text { Solo fumadores } \\
(n=4.785)\end{array}$} & \multicolumn{2}{|c|}{$\begin{array}{l}\text { Solo fumadores } \\
(n=4.785)\end{array}$} & \multicolumn{3}{|c|}{$\begin{array}{l}\text { Solo fumadores } \\
(\mathrm{n}=4.785)\end{array}$} & \multicolumn{2}{|c|}{$\begin{array}{l}\text { Solo fumadores } \\
(n=4.785)\end{array}$} & \multicolumn{2}{|c|}{$\begin{array}{l}\text { Solo fumadores } \\
(\mathrm{n}=4.785)\end{array}$} \\
\hline Variables capturando la clase social & \multicolumn{2}{|r|}{ Sí } & \multicolumn{2}{|c|}{ No } & \multicolumn{2}{|c|}{ Sí } & \multicolumn{3}{|c|}{ No } & \multicolumn{2}{|c|}{ Sí } & \multicolumn{2}{|r|}{ No } \\
\hline Variables relacionadas con la clase social & \multicolumn{2}{|c|}{ No } & \multicolumn{2}{|c|}{ Sí } & \multicolumn{2}{|c|}{ No } & & Sí & & & No & & Sí \\
\hline $\begin{array}{l}\text { Variables Independientes Principales } \\
\text {-Gradiente Socioeconómico- }\end{array}$ & OR & IC $95 \%$ & OR & IC $95 \%$ & OR & IC $95 \%$ & OR & IC 95 & $5 \%$ & OR & IC $95 \%$ & OR & IC $95 \%$ \\
\hline Clase social & & & & & & & & & & & & & \\
\hline Clase I (ref.) (0-1) & 1 & & & & 1 & & & & & 1 & & & \\
\hline Clase II (0-1) & 1,37 & $0,87 \quad 2,17$ & & & 1,13 & $0,851,51$ & & & & 0,93 & $0,51 \quad 1,69$ & & \\
\hline Clase III (0-1) & 1,48 * & $1,02 \quad 2,13$ & & & 1,24 & $0,98 \quad 1,57$ & & & & 0,87 & $0,54 \quad 1,39$ & & \\
\hline Clase IV (0-1) & 1,64 * & $1,09 \quad 2,46$ & & & 1,36 * & $1,061,75$ & & & & 0,83 & $0,511,36$ & & \\
\hline Clase V (0-1) & $1,99 * \star \star$ & $1,40 \quad 2,84$ & & & 1,34 * & $1,071,67$ & & & & 1,02 & $0,66 \quad 1,57$ & & \\
\hline Clase VI (0-1) & 1,68 * & $1,13 \quad 2,51$ & & & 1,50 ** & $1,17 \quad 1,93$ & & & & 1,12 & $0,70 \quad 1,79$ & & \\
\hline Nivel educativo & & & & & & & & & & & & & \\
\hline Educación básica (0-1) & & & 1,49 * & $1,012,20$ & & & $1,84^{\star \star \star}$ & 1,472 & 2,32 & & & 1,07 & $0,69 \quad 1,67$ \\
\hline Educación secundaria (0-1) & & & 1,48 * & $1,101,99$ & & & $1,66 * \star \star$ & 1,372 & 2,01 & & & 1,06 & $0,71 \quad 1,57$ \\
\hline Educación profesional (0-1) & & & 1,38 & $0,98 \quad 1,94$ & & & $1,55 * \star \star$ & 1,251 & 1,92 & & & 0,92 & $0,58 \quad 1,44$ \\
\hline Educación universitaria (ref.) $(0-1)$ & & & 1 & & & & 1 & & & & & 1 & \\
\hline Variables económico-laborales & & & & & & & & & & & & & \\
\hline Actividad principal & & & & & & & & & & & & & \\
\hline $\begin{array}{l}\text { Empresario o profesional } \\
\text { con asalariados }(0-1)\end{array}$ & & & 0,61 & $0,321,15$ & & & 0,91 & 0,611 & 1,35 & & & 0,74 & $0,38 \quad 1,47$ \\
\hline $\begin{array}{l}\text { Empresario sin asalariados o } \\
\text { trabajador independiente (ref.) (0-1) }\end{array}$ & & & 1 & & & & 1 & & & & & 1 & \\
\hline Funcionario (0-1) & & & 1,06 & $0,58 \quad 1,95$ & & & 0,89 & 0,631 & 1,27 & & & 0,53 & $0,26 \quad 1,08$ \\
\hline Trabajador indefinido (0-1) & & & 0,90 & $0,581,38$ & & & $0,72 * *$ & 0,560 & 0,91 & & & 0,57 * & $0,38 \quad 0,88$ \\
\hline Trabajador con contrato temporal (0-1) & & & 1,02 & $0,611,71$ & & & 0,80 & 0,591 & 1,07 & & & 0,75 & $0,43 \quad 1,29$ \\
\hline Otra situación de empleo (0-1) & & & 2,33 & $0,5310,27$ & & & 1,16 & 0,642 & 2,11 & & & 1,59 & $0,61 \quad 4,15$ \\
\hline Desempleado (0-1) & & & 0,97 & $0,61 \quad 1,53$ & & & 0,73 * & 0,570 & 0,94 & & & 0,74 & $0,47 \quad 1,15$ \\
\hline Estudiante (0-1) & & & 0,51 * & $0,280,94$ & & & $0,20 * \star \star$ & 0,120 & 0,33 & & & 0,27 & $0,06 \quad 1,18$ \\
\hline Jubilado o prejubilado (0-1) & & & 1,82 & $0,79 \quad 4,21$ & & & 0,78 & 0,541 & 1,14 & & & 0,44 * & $0,23 \quad 0,86$ \\
\hline Incapacitado para trabajar (0-1) & & & 0,77 & $0,351,73$ & & & 0,79 & 0,511 & 1,23 & & & 0,64 & $0,32 \quad 1,28$ \\
\hline Labores del hogar $(0-1)$ & & & 1,27 & $0,652,50$ & & & 0,81 & 0,571 & 1,15 & & & 0,65 & $0,31 \quad 1,36$ \\
\hline Otra situación de inactividad (0-1) & & & 0,91 & $0,117,77$ & & & 1,84 & $0,56 \quad 6$ & 6,05 & & & 1,96 & $0,59 \quad 6,48$ \\
\hline Seguro de salud & & & & & & & & & & & & & \\
\hline $\begin{array}{l}\text { Únicamente sanidad pública } \\
\text {-no mutualidades del Estado- (0-1) }\end{array}$ & & & 1,16 & $0,871,53$ & & & 0,90 & 0,761 & 1,07 & & & 0,94 & $0,661,32$ \\
\hline $\begin{array}{l}\text { Mutualidades del Estado o } \\
\text { seguro privado (ref.) }(0-1)\end{array}$ & & & 1 & & & & 1 & & & & & 1 & \\
\hline
\end{tabular}

Variables Independientes de Control

Variables sociodemográficas

\begin{tabular}{|c|c|c|c|c|c|c|c|c|c|c|c|c|c|c|c|c|c|c|}
\hline \multicolumn{19}{|l|}{ Sexo } \\
\hline Mujer (0-1) & 0,91 & 0,73 & 1,15 & 0,92 & 0,72 & 1,16 & 0,66 *** & 0,57 & 0,75 & $0,69 * \star *$ & 0,60 & 0,79 & $0,45^{* * *}$ & 0,34 & 0,60 & $0,46^{* \star *}$ & 0,34 & 0,62 \\
\hline Hombre (ref.) (0-1) & 1 & & & 1 & & & 1 & & & 1 & & & 1 & & & 1 & & \\
\hline \multicolumn{19}{|l|}{ Edad } \\
\hline $15-35$ años (ref.) (0-1) & 1 & & & 1 & & & 1 & & & 1 & & & 1 & & & 1 & & \\
\hline 36-50 años (0-1) & $1,94 * \star \star$ & 1,49 & 2,53 & $1,83 * \star \star$ & 1,39 & 2,40 & $1,98 * \star \star$ & 1,67 & 2,34 & $1,75^{\star * *}$ & 1,47 & 2,08 & $1,92 * *$ & 1,30 & 2,84 & $1,83 * *$ & 1,23 & 2,73 \\
\hline $51-65$ años (0-1) & $2,54 * \star \star$ & 1,77 & 3,64 & $2,15 * \star \star$ & 1,47 & 3,14 & $2,29 * \star \star$ & 1,86 & 2,81 & $1,92 * \star *$ & 1,54 & 2,39 & $2,55 * \star \star$ & 1,65 & 3,93 & $2,47 * \star \star$ & 1,58 & 3,87 \\
\hline Más de 65 años (0-1) & $3,15 * \star \star$ & 1,71 & 5,78 & 1,54 & 0,64 & 3,72 & $1,65 * \star$ & 1,22 & 2,21 & 1,33 & 0,88 & 2,00 & 1,79 & 0,98 & 3,26 & 2,37 * & 1,07 & 5,25 \\
\hline \multicolumn{19}{|l|}{ Estado civil } \\
\hline Soltero (ref.) (0-1) & 1 & & & 1 & & & 1 & & & 1 & & & 1 & & & 1 & & \\
\hline Casado (0-1) & 0,97 & 0,75 & 1,27 & 0,92 & 0,70 & 1,21 & 1,12 & 0,96 & 1,30 & 1,03 & 0,88 & 1,20 & 1,12 & 0,83 & 1,52 & 1,11 & 0,81 & 1,51 \\
\hline Separado (0-1) & 1,06 & 0,57 & 1,97 & 1,05 & 0,56 & 1,97 & 1,18 & 0,84 & 1,64 & 1,10 & 0,79 & 1,53 & 1,56 & 0,92 & 2,65 & 1,56 & 0,92 & 2,65 \\
\hline Divorciado (0-1) & 2,01 * & 1,12 & 3,62 & 1,94 * & 1,07 & 3,50 & $1,79 * \star \star$ & 1,39 & 2,31 & $1,72 \star \star \star *$ & 1,33 & 2,22 & 1,51 & 0,96 & 2,36 & 1,46 & 0,93 & 2,28 \\
\hline Viudo (0-1) & 2,46 * & 1,02 & 5,89 & 2,22 & 0,92 & 5,37 & 1,41 * & 1,01 & 1,97 & 1,29 & 0,92 & 1,81 & 1,35 & 0,72 & 2,54 & 1,49 & 0,78 & 2,83 \\
\hline Número de niños en el hogar (0-6) & 0,86 & 0,74 & 1,00 & 0,86 & 0,74 & 1,00 & 0,89 * & 0,81 & 0,97 & $0,88^{* *}$ & 0,80 & 0,97 & 0,88 & 0,72 & 1,07 & 0,86 & 0,70 & 1,04 \\
\hline \multicolumn{19}{|l|}{ Hábitos de vida saludable } \\
\hline $\begin{array}{l}\text { Horas semanales de ejercicio físico } \\
\text { en tiempo de ocio (0-50) }\end{array}$ & 0,96 ** & 0,94 & 0,99 & $0,96 * *$ & 0,94 & 0,99 & $0,95 * * *$ & 0,93 & 0,96 & $0,95 * * *$ & 0,93 & 0,96 & $0,90 * * *$ & 0,85 & 0,94 & $0,90 * * *$ & 0,85 & 0,94 \\
\hline $\begin{array}{l}\text { Índice de alimentación saludable } \\
(-13 \text { a } 25)\end{array}$ & $0,96 * \star *$ & 0,94 & 0,98 & $0,96 * * *$ & 0,94 & 0,98 & $0,96 * * *$ & 0,94 & 0,97 & $0,96 * \star \star$ & 0,94 & 0,97 & $0,95 * * *$ & 0,93 & 0,97 & $0,95 * * *$ & 0,93 & 0,98 \\
\hline $\begin{array}{l}\text { Consumo medio diario de gramos } \\
\text { de alcohol puro }(0-185,71)\end{array}$ & 1,00 & 0,99 & 1,01 & 1,00 & 0,99 & 1,01 & $1,01 * * \star$ & 1,01 & 1,02 & $1,01 * * *$ & 1,01 & 1,02 & $1,02 * * *$ & 1,01 & 1,03 & $1,02 * * *$ & 1,01 & 1,03 \\
\hline \multicolumn{19}{|l|}{ Estado de salud } \\
\hline Índice de Masa Corporal (1-4) & 0,93 & 0,80 & 1,08 & 0,92 & 0,79 & 1,06 & $1,15^{* *}$ & 1,06 & 1,25 & $1,13^{* *}$ & 1,04 & 1,23 & $1,58 * \star \star$ & 1,36 & 1,83 & $1,55 * \star \star$ & 1,34 & 1,81 \\
\hline \multicolumn{19}{|l|}{ Salud mental (últimos 12 meses) } \\
\hline $\begin{array}{l}\text { Enfermedad / problema de salud } \\
\text { mental (0-1) }\end{array}$ & 1,08 & 0,76 & 1,54 & 1,08 & 0,76 & 1,55 & $1,35 * *$ & 1,13 & 1,62 & $1,33^{* *}$ & 1,11 & 1,61 & $2,79 * \star \star$ & 2,09 & 3,71 & $2,79 * * *$ & 2,07 & 3,77 \\
\hline Mentalmente sano (ref.) (0-1) & 1 & & & 1 & & & 1 & & & 1 & & & 1 & & & 1 & & \\
\hline
\end{tabular}

Nota. ${ }^{*} p<0,05 ;{ }^{* \star} p<0,01 ;{ }^{* \star \star} p<0,001$. Todos los modelos incluyen también como variables de control las siguientes variables de tipo geográfico: lugar de nacimiento (variable categórica indicando si el participante ha nacido en España o fuera de España), tamaño del municipio (7 variables categóricas de menor a mayor población: 10 mil hab., $10-20$ mil hab., 20-50 mil hab., 50-100 mil hab., 100-500 mil hab. no capital de provincia, capital de provincia < 500 mil hab., > 500 mil hab.), comunidad (o ciudad) autónoma (19 variables categóricas correspondientes a las 17 CCAA y las 2 ciudades autónomas en España). Fuente. Elaboración propia con datos de la EESE-2014. 
de niños, mientras que aumenta en personas de entre 36 y 65 años (frente a personas de entre 15 y 35 años), en divorciados (frente a solteros), en la población con conductas menos saludables, y en personas con mayor IMC o con alguna enfermedad o problema de salud mental.

Finalmente, se analizan los resultados relacionados con la probabilidad de encontrar población fumadora a diario de más de 20 cigarrillos (frente a fumadora a diario de 20 o menos cigarrillos y fumadores ocasionales), que se muestran en el panel derecho de la Tabla 3. En relación con las variables asociadas al gradiente socioeconómico y, contrariamente a lo que ocurre para el resto de probabilidades analizadas durante este trabajo, los modelos 3A y 3B no muestran ningún efecto significativo de la clase social, la educación o el seguro de salud. En cuanto a la actividad principal (modelo 3B), sin embargo, frente a la situación de empresario sin asalariados o trabajador independiente, esta probabilidad disminuye para jubilados o prejubilados $(\mathrm{OR}=0,44)$ y trabajadores con contrato indefinido $(\mathrm{OR}=0,57)$.

En cuanto a las variables independientes de control, los modelos 3A y 3B muestran cómo esta probabilidad se reduce en mujeres y aumenta en personas de entre $36 \mathrm{y}$ 65 años (frente a personas de entre 15 y 35 años), en la población con peores hábitos, y en personas con mayor IMC o con alguna enfermedad o problema de salud mental.

\section{Discusión}

Para abordar las desigualdades en la prevalencia del tabaquismo, las intervenciones y las políticas sociosanitarias deben estar dirigidas a grupos con un mayor riesgo de prevalencia., siendo la identificación de estos grupos de riesgo el principal objetivo de este trabajo.

Los resultados obtenidos confirman la relación entre el gradiente socioeconómico tanto con la prevalencia como con el nivel de consumo en España, lo que es coherente con las predicciones del modelo epidemiológico de López et al. (1994) para la difusión de la epidemia en los grupos más desfavorecidos y con la propia literatura académica internacional que documenta esta asociación (Schaap et al., 2008; White et al., 2016). Estos resultados son robustos a su identificación bien mediante las variables capturando las clases sociales, o bien a través del nivel educativo y las variables económico-laborales.

Más concretamente, en relación con la clase social, se observa cómo tanto la prevalencia como el nivel de consumo de cigarrillos aumenta en los grupos de inferior clase social. En este sentido, la clase social (basada en la ocupación) puede indicar diferencias entre los trabajadores en el entorno laboral y las relaciones sociales en el trabajo, que pueden generar importantes diferencias en relación con el tabaquismo debido a la heterogeneidad existente dentro de cada grupo en cuanto a actitudes, normas sociales y apoyo social (Sorensen, Barbeau, Hunt y Emmons, 2004).

En cuanto al nivel educativo, se observa en concreto cómo la educación universitaria no solo está asociada con una menor prevalencia en el consumo sino, dentro de la población de fumadores, con un menor nivel de consumo. En relación con este resultado, también se observa cómo la actividad principal de estudiante está asociada con una menor prevalencia y el consumo, lo que sugiere la importancia de los procesos educativos formales en la lucha contra esta epidemia. No en vano, el nivel educativo es el factor vinculado al gradiente socioeconómico más utilizado en el conjunto de trabajos que analizan la asociación entre situación socioeconómica y tabaquismo (Schaap et al., 2008). En la práctica, las personas con más educación obtienen mejores resultados en casi todas las dimensiones de la salud, adoptan comportamientos más saludables y viven más tiempo (Maralani, 2014).

También resulta interesante el resultado asociado a aquellos participantes en situación de desempleo, que presentan una elevada prevalencia combinada con niveles de consumo más reducidos. Esta dualidad parece indicativa de un doble efecto. De un lado, la situación de desempleo no solo podría considerarse un suceso generador de estrés, lo que está asociado con la recaída en el hábito tabáquico (McKee, Maciejewski, Falba y Mazure, 2003), sino que ubica al individuo dentro de un colectivo más desfavorecido y vulnerable, en el que la presencia del tabaquismo es mayor (Falba, Teng, Sindelar y Gallo, 2005; Okechukwu, Bacic, Cheng y Catalano, 2012). Esta mayor prevalencia observada en el grupo de desempleados es coherente con lo observado para aquellos participantes que tienen como única modalidad de seguro médico la sanidad pública (y, por tanto, una peor situación económica), cuya prevalencia también es mayor. En este sentido, existe evidencia que asocia menores niveles de ingresos por trabajo a la población de fumadores (Levine, Gustafson y Velenchik, 1997). Del otro, la menor capacidad adquisitiva de estos grupos puede hacer que las personas simplemente reduzcan su nivel de consumo (Falba et al., 2005). En el caso de los desempleados en España, en concreto, coexisten no solo las propias dificultades financieras asociadas con la falta de empleo, sino también los efectos de la crisis de 2008 y el elevado precio de los cigarrillos.

Esta situación permite desarrollar algunos razonamientos interesantes. En primer lugar, parece urgente la necesidad de incorporar al colectivo de desempleados en planes específicos de prevención y abandono del hábito. En segundo lugar, las políticas de empleo desarrolladas por los distintos servicios públicos no solo generan un efecto directo en términos de la reducción del desempleo sino que podrían tener un importante efecto indirecto en la lucha contra el tabaquismo. En tercer lugar, las subidas 
impositivas a los productos del tabaco parecen generar mayores disminuciones en los niveles de consumo de los colectivos de menor renta, esto es, aquellos que muestran una mayor prevalencia, lo que sugiere la conveniencia de estas medidas. En otras palabras, se observa una mayor elasticidad precio de la demanda de cigarrillos en los grupos socioeconómicos más vulnerables, lo que es coherente con la evidencia existente para otros países (Colman y Remler, 2008; Nargis, Fong, Chaloupka y Li, 2014). En ese sentido, sin embargo, las últimas subidas impositivas han hecho que España deje de ser un país de tránsito del mercado ilegal a un mercado de destino, sobre todo en regiones como Andalucía y Extremadura donde el nivel de desempleo es precisamente mayor (Calderoni, Angelini, Mancuso y Rotondi, 2014). Por tanto, estas subidas de impuestos deben venir acompañadas de una mayor presión contra la evasión fiscal y el contrabando que evite que se produzca un efecto sustitución en el consumo de productos legales hacia productos ilícitos.

$\mathrm{Al}$ poner el foco en la población trabajadora, se observan diversos sectores donde la prevalencia en el hábito es más reducida, algunos de ellos asociados a mayores niveles educativos como el sector educativo, el de actividades profesionales, científicas y técnicas, el de administración pública y defensa o el de actividades artísticas, recreativas y de entretenimiento. Lo contrario se observa, sin embargo, en otros sectores como la hostelería, la construcción o el transporte y almacenamiento. Estos resultados son coherentes con la evidencia disponible en la literatura internacional sobre una mayor prevalencia y nivel de consumo de tabaco en los trabajadores manuales (o de cuello azul) frente a los trabajadores de oficina (o de cuello blanco) (Okechukwu et al., 2012). Se deriva por tanto la necesidad de extremar la vigilancia en los trabajadores de sectores concretos, diseñando para ellos programas más sistemáticos de control del tabaquismo. En este sentido, el propio lugar de trabajo se ha revelado como un contexto efectivo para intervenciones de control del hábito en trabajadores manuales (Cahill y Lancaster, 2014). Sin embargo, la propia naturaleza de muchos trabajos manuales hace que los trabajadores cambien de empleador con frecuencia, lo que dificulta llegar a ellos a través de estos programas (Okechukwu et al., 2012). Además, son precisamente estos sectores los más sensibles a las fluctuaciones económicas lo que lleva a sus trabajadores a alternar de forma recurrente situaciones de empleo y desempleo (tal y como puede observarse en la actual crisis debida al COVID-19, especialmente en el sector de la hostelería). Esta alternancia coloca a estos trabajadores en una situación de doble vulnerabilidad en términos de su hábito tabáquico. De un lado, la existencia de una mayor prevalencia y nivel de consumo en situaciones de pérdida de empleo (Montgomery, Cook, Bartley y Wadsworth, 1998) y, del otro, la existencia de un mayor nivel de consumo en situaciones económicas recesivas (Okechukwu et al., 2012).

Estos resultados en su conjunto no solo permiten identificar de forma más precisa los grupos de riesgo en la lucha contra el tabaquismo en España, sino que ponen de manifiesto la necesidad de recopilar más evidencias que favorezcan un mejor tratamiento de la dependencia en poblaciones especiales, como son los colectivos con menor nivel socioeconómico (Fagan et al., 2004).

Este estudio también incluye en el análisis otras variables de control que guardan relación con el tabaquismo, tales como características sociodemográficas, hábitos de vida saludables y estado de salud. Del análisis de los resultados obtenidos en relación con estas variables también se desprenden algunos razonamientos de interés.

En primer lugar, este trabajo pone de manifiesto algunos factores demográficos que caracterizan al consumidor de cigarrillos en España. En concreto, los varones, las personas de entre 36 y 50 años, las personas divorciadas, y las personas cuyos hijos no viven en el hogar presentan tanto una mayor prevalencia como intensidad en el consumo de cigarrillos. En este sentido, si bien los resultados asociados al sexo y edad del fumador son muy habituales en los estudios epidemiológicos (Pinilla y Abásolo, 2017; Leal-López, Sánchez-Queija y Moreno, 2019; RodríguezMuñoz, Carmona-Torres, Hidalgo-Lopezosa, Cobo-Cuenca y Rodríguez-Borrego, 2019), la evidencia obtenida en relación con el estado civil y el número de hijos en el hogar es más novedosa y podría ser de interés de cara a un mejor diseño de programas de control de tabaco para colectivos específicos. En particular, estos resultados sugieren una relación con el conjunto de trabajos que identifican una asociación positiva entre el hábito tabáquico y factores como la soledad y el afecto negativo (véase Dyal y Valente, 2015, para una revisión).

En segundo lugar, se observa una asociación robusta entre los hábitos de vida saludables y la menor prevalencia e intensidad en el consumo de cigarrillos, lo que es coherente con la literatura académica existente, si bien dentro de esta última predominan los trabajos focalizados en subgrupos concretos de población como adolescentes (Rodríguez-García, López Villalba, López-Miñarro y García-Cantó, 2013), grupos marginales (Watanabe et al., 2013) o mujeres embarazadas (Ino, Shibuya, Saito e Inaba, 2011). Por tanto, los resultados presentados en este trabajo contribuyen a esta literatura mostrando la existencia de esta asociación virtuosa 'hábitos de vida saludable - menor hábito tabáquico' para el total de la población, lo cual es más infrecuente en los trabajos académicos (el trabajo de Agudo et al., 2004 es una excepción). Expresado en forma compacta, la práctica habitual de ejercicio físico, una correcta alimentación y nutrición y el consumo responsable de alcohol no solo son conductas altamente recomendables por sus enormes beneficios físicos y psíquicos (Woodcock, 
Franco, Orsini y Roberts, 2011), sino que su promoción se revela como un aliado indispensable en el diseño de planes contra la epidemia tabáquica por parte de las autoridades sanitarias.

En tercer lugar, este análisis presenta otras evidencias valiosas de cara a su contribución al diseño de programas de control de tabaco para colectivos específicos como las que relacionan el consumo de cigarrillos y el estado de salud, capturado este a través del IMC y el estado de salud mental. Respecto al IMC, los resultados de este trabajo muestran cómo los fumadores presentan valores del índice inferiores a los que presentan los no fumadores. Sin embargo, los resultados también muestran cómo a medida que aumenta el nivel de consumo, también aumenta el valor del índice. En este sentido, ambas asociaciones son coherentes con la evidencia disponible (Chiolero, Faeh, Paccaud y Cornuz, 2008). En concreto, la reducción de apetito y el mayor gasto de energía asociado a la nicotina parece explicar la asociación negativa entre la prevalencia en el consumo y el valor del índice. Por su parte, los mayores niveles de consumo parecen estar asociados con un conjunto de factores de riesgo que coexisten (vida sedentaria, alimentación desordenada y exceso en el consumo de alcohol), y que podrían explicar el aumento de peso. Poniendo el foco en la salud mental, este trabajo presenta evidencias que muestran una relación positiva entre el consumo de cigarrillos y tener alguna enfermedad o problema de salud mental, lo que es coherente con los resultados existentes en la literatura epidemiológica, que relacionan el consumo de tabaco y problemas como depresión o ansiedad (Mykletun, Overland, Aarø, Liabø y Stewart, 2008).

Este trabajo no está exento de limitaciones, siendo los datos de sección cruzada disponibles en la EESE-2014 probablemente la más importante. Así, el hecho de que la información disponible haga referencia a un único periodo no permite establecer relaciones causa efecto, sino tan solo asociaciones estadísticas. En otras palabras, no es posible, a la luz de esta evidencia, realizar afirmaciones del tipo, un fumador lo es por su situación laboral, económica, familiar o simplemente por su estilo de vida. Además, los datos de este estudio no permiten distinguir el cigarrillo habitual de la picadura de liar, que sería interesante per se, ni incorporar otros productos del tabaco como puros o pipas, por el reducido número de observaciones en la muestra, o pipas de agua o cigarrillos electrónicos, por la exclusión de estos productos del cuestionario. Por su parte, la exploración de posibles efectos conjuntos entre las distintas variables asociadas al gradiente socioeconómico también ha quedado fuera de los límites del presente trabajo por brevedad y foco. Otras extensiones naturales de este estudio sería el uso de los datos de EESE-2019 (todavía no disponibles a fecha de escritura), lo que permitiría conocer cómo ha evolucionado el hábito tabáquico en
España en el último quinquenio, o la ampliación del marco de referencia a otros países también participantes en la Encuesta Europea de Salud, lo que permitiría la perspectiva comparada con los países del entorno socioeconómico.

\section{Reconocimientos}

Este estudio forma parte de la tesis doctoral de Juan Manuel Martín Álvarez, que se elaboró bajo la dirección de José María Millán y Antonio Golpe. Queremos expresar nuestra gratitud a Fernando Millán (Universidad de Sevilla) y a los evaluadores anónimos por sus comentarios y sugerencias. Este trabajo se ha beneficiado de la ayuda financiera recibida de la Junta de Andalucía a través del Grupo de Investigación SEJ-487 (Spanish Entrepreneurship Research Group [SERG]), de la Universidad de Huelva a través de la Estrategia de Política de Investigación y Transferencia (EPIT), de la Universidad Internacional de La Rioja a través de las ayudas a la publicación del Vicerrectorado de Investigación, y de la Universidad de Antioquia a través del Grupo de Microeconomía Aplicada de la Facultad de Ciencias Económicas.

\section{Conflicto de intereses}

Los autores del presente estudio declaran que no poseen conflicto de intereses.

\section{Referencias}

Agudo, A., Pera, G., Rodríguez, M., Quirós, J. R., Navarro, C., Martínez, C.,... González, C. A. (2004). Changes in smoking habits in adults: Results from a prospective study in Spain. Annals of Epidemiology, 14, 235-243. doi:10.1016/s1047-2797(03)00245-x.

Almeida, A., Golpe, A. A., Iglesias, J. y Martín-Álvarez, J. M. (2021). The price elasticity of cigarettes: New evidence from Spanish regions, 2002-2016. Nicotine $\mathcal{E}$ Tobacco Research, 23, 48-56. doi:10.1093/ntr/ntaa131.

Calderoni, F., Angelini, M., Mancuso, M. y Rotondi, M. (2014). Informe sobre comercio ilícito de productos del tabaco $N^{o} 7$ - España. Transcrime -Joint Research Centre on Transnational Crime. Trento: Università degli Studi di Trento. Recuperado de http://www.transcrime.it/ wp-content/uploads/2014/10/Factbook-Spain-Esp.pdf.

Cahill, K. y Lancaster, T. (2014). Workplace interventions for smoking cessation (Review). Cochrane Database of Systematic Reviews, Issue 2, CD003440. doi:10.1002/14651858.CD003440.pub4.

Chiolero, A., Faeh, D., Paccaud, F. y Cornuz, J. (2008). Consequences of smoking for body weight, body fat distribution, and insulin resistance. American Journal of Clinical Nutrition, 87, 801-809. doi:10.1093/ ajcn/87.4.801. 
Colman, G. J. y Remler, D. K. (2008). Vertical equity consequences of very high cigarette tax increases: If the poor are the ones smoking, how could cigarette tax increases be progressive?. Journal of Policy Analysis and Management, 27, 376-400. doi:10.1002/pam.20329.

Dyal, S. R. y Valente, T. W. (2015). A systematic review of loneliness and smoking: Small effects, big implications. Substance Use Eं Misuse, 50, 1697-1716. doi:10.3109/108 26084.2015.1027933.

European Commission (2012). Special Eurobarometer 385. Attitudes of Europeans towards tobacco. Bruselas: E. C., Directorate-General Health and Consumers. Recuperado de https://ec.europa.eu/health//sites/ health/files/tobacco/docs/eurobaro_attitudes_ towards_tobacco_2012_en.pdf.

European Commission (2014). Special Eurobarometer 429. Attitudes of Europeans towards tobacco and electronic cigarettes. Bruselas: E. C., Directorate-General for Health and Food safety. Recuperado de https://ec.europa. $\mathrm{eu} /$ commfrontoffice/publicopinion/archives/ebs/ ebs_429_en.pdf.

European Commission (2017). Special Eurobarometer 458. Attitudes of Europeans towards tobacco and electronic cigarettes. Bruselas: E. C., Directorate-General for Health and Food safety. Recuperado de https://ec.europa. $\mathrm{eu} /$ commfrontoffice/publicopinion/index.cfm/ ResultDoc/download/DocumentKy/79002.

Fagan, P., King, G., Lawrence, D., Sallie, A. P., Robinson, R. G., Banks, D.,.. Grana, R. (2004). Eliminating tobacco-related health disparities: Directions for future research. American Journal of Public Health, 94, 211-217. doi:10.2105/AJPH.94.2.211.

Falba, T., Teng, H. M., Sindelar, J. L. y Gallo, W. T. (2005). The effect of involuntary job loss on smoking intensity and relapse. Addiction, 100, 1330-1339. doi:10.1111/ j.1360-0443.2005.01150.x.

Ino, T., Shibuya, T., Saito, K. e Inaba, Y. (2011). Relationship between body mass index of offspring and maternal smoking during pregnancy. International Journal of Obesity, 36, 554-558. doi:10.1038/ijo.2011.255.

Leal-López, E., Sánchez-Queija, I. y Moreno, C. (2019). Trends in tobacco use among adolescents in Spain (2002-2018). Adicciones, 31, 289-297. doi:10.20882/ adicciones. 1111.

Levine, P. B., Gustafson T. A. y Velenchik, A. D. (1997). More bad news for smokers? The effects of cigarette smoking on wages. Industrial and Labor Relations Review, 50, 493-509. doi:10.1177/001979399705000307.

López, A. D., Collishaw, N. E. y Piha, T. (1994). A descriptive model of the cigarette epidemic in developed countries. Tobacco Control, 3, 242-247.

López-Nicolás, Á., Cobacho, M. B. y Fernández, E. (2013). The Spanish tobacco tax loopholes and their consequences. Tobacco Control, 22, e21-e24. doi:10.1136/ tobaccocontrol-2011-050344.

Macintyre, S. (1997). The black report and beyond what are the issues? Social Science $\mathcal{E}$ Medicine, 44, 723-745. doi:10.1016/S0277-9536(96)00183-9.

Maralani, V. (2014). Understanding the links between education and smoking. Social Science Research, 48, 20-34. doi:10.1016/j.ssresearch.2014.05.007.

Martín-Álvarez, J. M., Golpe, A. A., Iglesias, J. e Ingelmo, R. (2020). Price and income elasticities of demand for cigarette consumption: What is the association of price and economic activity with cigarette consumption in Spain from 1957 to 2016? Public Health, 185, 275-282. doi:10.1016/j.puhe.2020.05.059.

McKee, S. A., Maciejewski, P. K., Falba, T. y Mazure, C. M. (2003). Sex differences in the effects of stressful life events on changes in smoking status. Addiction, 98, 847855. doi:10.1046/j.1360-0443.2003.00408.x.

Ministerio de Sanidad Servicios Sociales e Igualdad, Instituto Nacional de Estadística. (2015). Encuesta Europea de Salud en España EESE-2014. Madrid: Ministerio de Sanidad, Servicios Sociales e Igualdad. Recuperado de http://www.ine.es.

Ministerio de Sanidad Servicios Sociales e Igualdad, Instituto Nacional de Estadística. (2018). Encuesta Nacional de Salud. España ENSE-2017. Madrid: Ministerio de Sanidad, Servicios Sociales e Igualdad. Recuperado de http://www.ine.es.

Montgomery, S. M., Cook, D. G., Bartley, M. y Wadsworth, M. E. J. (1998). Unemployment, cigarette smoking, alcohol consumption and body weight in young British men. European Journal of Public Health, 8, 21-27. doi:10.1093/eurpub/8.1.21.

Mykletun, A., Overland, S., Aarø, L. E., Liabø, H. -M. y Stewart, R. (2008). Smoking in relation to anxiety and depression: Evidence from a large population survey: The HUNT study. European Psychiatry, 23, 77-84. doi:10.1016/j.eurpsy.2007.10.005.

Nargis, N., Fong, G.T., Chaloupka, F.J. y Li, Q. (2014). The choice of discountbrand cigarettes:Acomparativeanalysis of International Tobacco Control Surveys in Canada and the USA (2002-2005). Tobacco Control, 23 (Supl. 1), i86-i96. doi:10.1136/tobaccocontrol-2012-050851.

Okechukwu, C., Bacic, J., Cheng, K. W. y Catalano, R. (2012). Smoking among construction workers: The non-linear influence of the economy, cigarette prices, and antismoking sentiment. Social Science $\mathcal{E}$ Medicine, 75, 1379-1386. doi:10.1016/j.socscimed.2012.05.035.

Pinilla, J. y Abásolo, I. (2017). The effect of policies regulating tobacco consumption on smoking initiation and cessation in Spain: Is it equal across socioeconomic groups?. Tobacco Induced Diseases, 15, 8. doi:10.1186/ s12971-016-0109-4. 
Rodríguez-García, P. L., López Villalba, F. J., LópezMiñarro, P. A. y García-Cantó, E. (2013). Práctica de ejercicio físico y su relación con el consumo de tabaco en adolescentes. Diferencias entre géneros. Adicciones, 25, 29-36. doi:10.20882/adicciones.25.1.

Rodríguez-Muñoz, P.M., Carmona-Torres, J.M., HidalgoLopezosa, P., Cobo-Cuenca, A.I. y Rodríguez-Borrego, M.A. (2019). Evolution of alcohol and tobacco consumption in young people in Spain, after the law 42/2010 against smoking: 2011-2014. Adicciones, 31, 274283. doi:10.20882/adicciones.1035.

Sarkar, B. K., Shahab, L., Arora, M., Ahluwalia, J. S., Reddy, K. S. y West, R. (2017). The social gradient in tobacco use does not generalize to low-income urban communities in India: Findings from a census survey. Nicotine E̋ Tobacco Research, 19, 1516-1520. doi:10.1093/ ntr/ntw214.

Schaap, M. M., Van Agt, H. y Kunst, A. (2008). Identification of socioeconomic groups at increased risk for smoking in European countries: Looking beyond educational level. Nicotine E Tobacco Research, 10, 359369. doi:10.1080/14622200701825098.

Sorensen, G., Barbeau, E. M., Hunt, M. K. y Emmons, K. (2004). Reducing social disparities in tobacco use: A social-contextual model for reducing tobacco use among blue-collar workers. American Journal of Public Health, 94, 230-239. doi:10.2105/AJPH.94.2.230.

Stringhini, S., Sabia, S., Shipley, M., Brunner, E., Nabi, H., Kivimaki, M. y Singh-Manoux, A. (2010). Association of socioeconomic position with health behaviors and mortality. JAMA, 303, 1159-1166. doi:10.1001/ jama.2010.297.

Watanabe, I., Shigeta, M., Inoue, K., Matsui, D., Ozaki, E., Kuriyama, N.,... Watanabe, Y. (2013). Personal factors associated with smoking among marginalized and disadvantaged youth in Japan. International Journal of Behavioral Medicine, 20, 504-513. doi:10.1007/s12529-0129268-8.

White, T. J., Redner, R., Bunn, J. Y. y Higgins, S. T. (2016). Do socioeconomic risk factors for cigarette smoking extend to smokeless tobacco use? Nicotine $\mathcal{E}$ Tobacco Research, 18, 869-873. doi:10.1093/ntr/ntv199.

Woodcock, J., Franco, O. H., Orsini, N. y Roberts, I. (2011). Non-vigorous physical activity and all-cause mortality: Systematic review and meta-analysis of cohort studies. International Journal of Epidemiology, 40, 121-138. doi:10.1093/ije/dyq104. 